\title{
Simultaneous epigenetic perturbation and genome imaging reveal distinct roles of H3K9me3 in chromatin architecture and transcription
}

Ying Feng ${ }^{1+}$, Yao Wang ${ }^{2,3+}$, Xiangnan Wang ${ }^{4}$, Xiaohui He ${ }^{4}$, Chen Yang ${ }^{5}$, Ardalan Naseri ${ }^{6}$, Thoru Pederson ${ }^{7}$, Jing Zheng ${ }^{5}$, Shaojie Zhang ${ }^{6}$, Xiao Xiao ${ }^{8}$, Wei Xie ${ }^{2,3^{*}}$ and Hanhui Ma ${ }^{4^{*}}$

\author{
*Correspondence: xiewei121@ \\ tsinghua.edu.cn; mahh@ \\ shanghaitech.edu.cn \\ ${ }^{+}$Ying Feng and Yao Wang \\ contributed equally to this work. \\ ${ }^{2}$ Center for Stem Cell Biology and \\ Regenerative Medicine, MOE Key \\ Laboratory of Bioinformatics, School \\ of Life Sciences, Tsinghua University, \\ Beijing, China \\ ${ }^{4}$ School of Life Science and \\ Technology, ShanghaiTech \\ University, Beijing, China \\ Full list of author information is \\ available at the end of the article
}

\begin{abstract}
Introduction: Despite the long-observed correlation between H3K9me3, chromatin architecture, and transcriptional repression, how H3K9me3 regulates genome higherorder organization and transcriptional activity in living cells remains unclear.

Result: Here, we develop EpiGo (Epigenetic perturbation induced Genome organization)-KRAB to introduce H3K9me3 at hundreds of loci spanning megabases on human chromosome 19 and simultaneously track genome organization. EpiGoKRAB is sufficient to induce genomic clustering and de novo heterochromatin-like domain formation, which requires SETDB1, a methyltransferase of H3K9me3. Unexpectedly, EpiGo-KRAB-induced heterochromatin-like domain does not result in widespread gene repression except a small set of genes with concurrent loss of H3K4me3 and H3K27ac. Ectopic H3K9me3 appears to spread in inactive regions but is largely restricted from transcriptional initiation sites in active regions. Finally, $\mathrm{Hi}-\mathrm{C}$ analysis showed that EpiGo-KRAB reshapes existing compartments mainly at compartment boundaries.
\end{abstract}

Conclusions: These results reveal the role of $\mathrm{H} 3 \mathrm{~K} 9 \mathrm{me} 3$ in genome organization could be partially separated from its function in gene repression.

\section{Background}

Human genome is organized in a hierarchy manner from kilobase to megabase scales such as nucleosome, loops, topologically associated domains (TADs), and A/B compartments [1-4]. It has been proposed that the loop extrusion drives TAD formation [5]. On the other hand, liquid-liquid phase separation is suggested to mediate genome compartmentalization [5, 6]. For example, heterochromatin protein HP1 $\alpha$ undergoes liquid-liquid demixing suggesting a role of phase separation in heterochromatin domain formation [7-9]. Heterochromatin drives compartmentalization in the inverted nuclei of rods in nocturnal mammals [10]. The co-segregated compartments often share similar

(c) The Author(s). 2020 Open Access This article is licensed under a Creative Commons Attribution 4.0 International License, which permits use, sharing, adaptation, distribution and reproduction in any medium or format, as long as you give appropriate credit to the original author(s) and the source, provide a link to the Creative Commons licence, and indicate if changes were made. The images or other third party material in this article are included in the article's Creative Commons licence, unless indicated otherwise in a credit line to the material. If material is not included in the article's Creative Commons licence and your intended use is not permitted by statutory regulation or exceeds the permitted use, you will need to obtain permission directly from the copyright holder. To view a copy of this licence, visit http://creativecommons.org/licenses/by/4.0/. The Creative Commons Public Domain Dedication waiver (http://creativecommons.org/publicdomain/zero/1.0/) applies to the data made available in this article, unless otherwise stated in a credit line to the data. 
chromatin states such as histone marks [11]. Despite the widely observed correlations [11], how the epigenetic modifications regulates genome organization, particularly in living cells, remains unclear. Direct visualization of chromatin structures in cell nucleus is still challenging. Multiplexed DNA FISH, OligoSTORM, Hi-M, or ORCA has been applied to trace DNA folding [12-16] in fixed cells. On the other hand, CRISPR-based imaging provides a versatile and powerful tool to track chromatin topology in live cells in real time $[17,18]$.

Here we develop a CRISPR-based EpiGo-KRAB system to investigate the effect of ectopic H3K9me3 on genome organization and transcription in living cells. We show EpiGo-KRAB of a large chromatin domain induces de novo heterochromatin-like domain formation and reshapes local compartmentalization. Surprisingly, EpiGo-KRABinduced heterochromatin-like domain does not coccurently result in global gene repression. We believe that this system should be applicable for other epigenetic perturbations.

\section{Results}

\section{Establishment of EpiGo-KRAB}

To investigate how H3K9me3 regulates genome architecture and gene expression in living cells, here we developed a CRISPR-based system, namely EpiGo (Epigenetic perturbation induced Genome organization)-KRAB (Fig. 1a). The EpiGo-KRAB system allows to epigenetic manipulation of defined regions and visualizing the subsequent spatiotemporal dynamics of these loci. First, we utilized dCas9-KRAB, which deposits loci-specific epigenetic modifications [19, 20], and fluorescent guide RNAs from the CRISPRainbow system (sgRNA-2XPP7 and PCP-GFP) for DNA visualization [21]. We intended to alter the epigenetic states from kilobases to megabase scales, to study the role of epigenetic modification in genome organization at different scales. By mining the chromosome-specific repeats across megabases of human genome, we found a repeat class which consists of 836 copies of CRISPR target sites spanning $\sim 17$ megabases at the q-arm of chromosome 19, and we dubbed it C19Q as EpiGo-KRAB targets for the following studies (Fig. 1a and Table S1). C19Q can be visualized by co-expression of dCas9-KRAB, sgRNA2XPP7, and PCP-GFP. We termed it as C19Q-KRAB. C19Q-KRAB could presumably recruit SETDB1 [22, 23] which deposits loci-specific H3K9me3 [24]. HP1 $\alpha$ could then interact with loci or regions with H3K9me3 [25, 26]. Thus, we expect that EpiGo of C19Q will allow us to track the changes of genome organization upon epigenetic alterations in living cells. To further track the dynamic interaction between EpiGo-mediated H3K9me3 and HP1 $\alpha$ condensates, HaloTag was knockedin at the C-terminus of HP1 $\alpha$ by CRISPR-Cas9 system. This U2OS-HP1 $\alpha$-HaloTag cell line was then used to generate cell lines stably expressed C19Q-sgRNA-2XPP7, PCP-GFP and dCas9 or dCas9-KRAB, resulting in U2OS-EpiGo-Control or U2OSEpiGo-KRAB cells for direct visualization of C19Q upon ectopic H3K9me3 modifications (Fig. 1b, c). Finally, ChIP-seq analysis confirmed that most target site of C19Q successfully acquired ectopic H3K9me3 (Fig. S1 and Fig. 1d). These data demonstrate that we have successfully induced large scale epigenetic alterations using EpiGo-KRAB. 
A

EpiGo-KRAB for simultaneous epigenetic perturbation and genome imaging

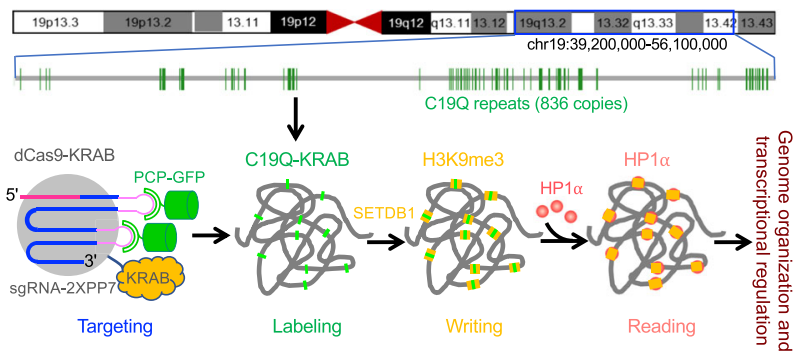

B

C

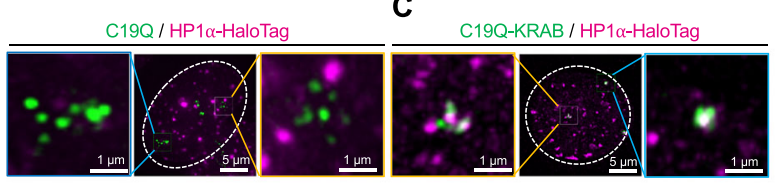

D

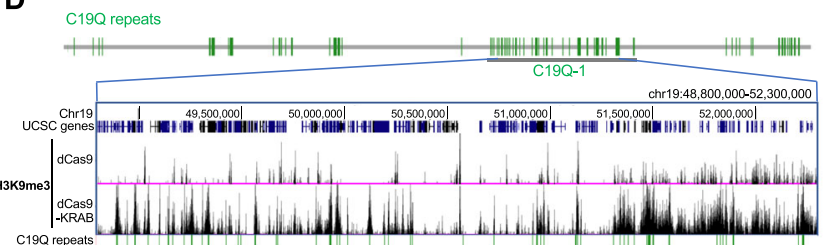

Fig. 1 A CRISPR-based EpiGo-KRAB system to track genome organization upon epigenetic modification. a A scheme of the EpiGo system. EpiGo (Epigenetic perturbation induced Genome organization) system consists of dCas9-KRAB, PCP-GFP, and sgRNA-2XPP7 targeting to C19Q repeats, which contain 836 copies of target sites on the q-arm of chromosome 19. C19Q-KRAB will recruit SETDB1 and induce H3K9

trimethylation at each target sites. $\mathrm{H} 3 \mathrm{~K} 9 \mathrm{me} 3$ regions will recruit $\mathrm{HP} 1 \mathrm{a}$ and induce genome organization. $\mathbf{b}$ CRISPR-based imaging of C19Q in presence of PCP-GFP, C19Q-sgRNA-2XPP7, and dCas9, marked with GFPC19Q. The HP1a-HaloTag stable cell lines was used to examine the colocalization between C19Q (green) and HP1a (magenta). $\mathbf{c}$ CRISPR imaging with the same conditions as $\mathbf{b}$ except that dCas9 was replaced by dCas9-KRAB and marked with GFP-C19Q-KRAB. d H3K9me3 state of EpiGo-Control (dCas9) and EpiGo-KRAB (dCas9-KRAB) cell lines. ChIP-seq of H3K9me3 was performed in these two cell lines. C19Q-1 region (chr19: $48,800,000-52,300,000$ ) is chosen to show the difference of H3K9me3 state between EpiGo-Control and EpiGo-KRAB cell lines

\section{EpiGo-KRAB induces genomic clustering adjacent to HP1a condensates}

We then sought to examine the spatiotemporal dynamics of $\mathrm{C} 19 \mathrm{Q}$ regions upon EpiGo-KRAB-mediated H3K9me3. As shown in Fig. 2a, discrete foci of C19Q (green) were visible by CRISPR labeling in U2OS-EpiGo-Control (dCas9) cells, which barely colocalize with HP1 $\alpha$ (magenta) over time. However, in EpiGo-KRAB cell lines (dCas9KRAB), C19Q loci dynamically interacted with HP1 $\alpha$ condensates and clustered around HP1 $\alpha$ condensates. Eventually, adjacent HP1 $\alpha$ condensates coalesced together. To investigate how HP1 $\alpha$ mediates local compaction of genomic regions upon EpiGo-KRAB induction (Fig. 2b), structured illumination microscopy (3D-SIM) was used to acquire high resolution imaging of C19Q and HP1 $\alpha$. We found that C19Q foci localized nearby HP1 $\alpha$ condensates in EpiGo-KRAB (dCas9-KRAB) cell lines (Fig. 2b). Quantitative analysis confirmed that $\mathrm{C} 19 \mathrm{Q}$ foci were not clustered in EpiGo-Control cells, while $90 \%$ of C19Q foci showed clustering in EpiGo-KRAB cells (Fig. 2c). In sum, these results suggest that EpiGo-KRAB can induce genome reorganization through a dynamic and stepwise process.

It was reported that dCas9-KRAB-mediated H3K9me3 deposition via histone methyltransferase SETDB1 [23, 27]. To test whether SETDB1 is essential for EpiGo-KRAB- 


\section{A}

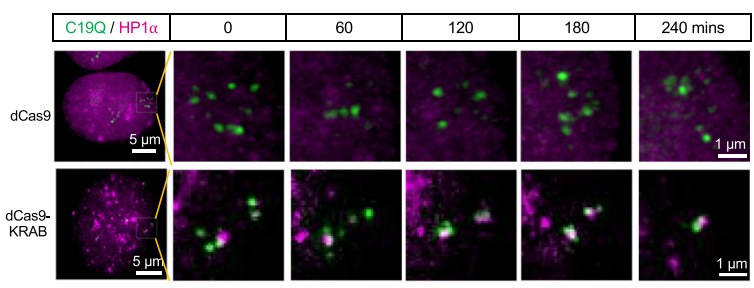

B

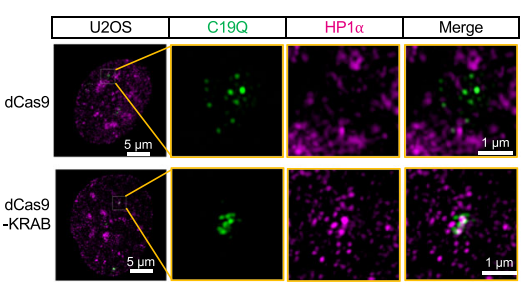

C

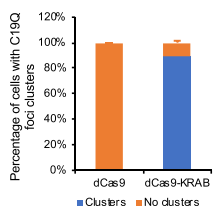

E

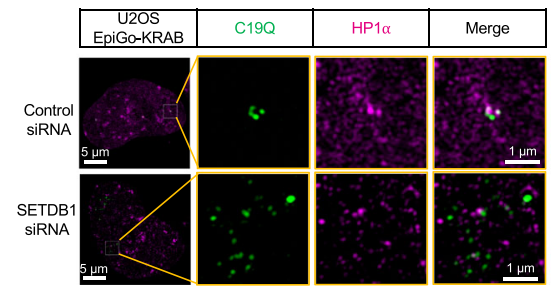

D
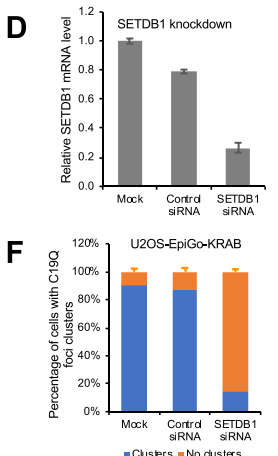

Fig. 2 SETDB1 is required for EpiGo-KRAB-mediated genomic clustering. a Live cell tracking of C19Q dynamics in EpiGo-Control (dCas9) and EpiGo-KRAB (dCas9-KRAB) cell lines. These cell lines were stably expressed PCP-GFP, C19Q-sgRNA-2XPP7, and dCas9 or dCas9-KRAB. The HaloTag was knocked-in at the Cterminus of HP1a and used to examine the colocalization between C19Q (green) and HP1a (magenta). The dCas9 or dCas9-KRAB was induced to express $24 \mathrm{~h}$ before tracking. The dynamics of C19Q (green) and HP1a (magenta) were tracked for 240 mins. Images were captured every 30 mins. b 3D-SIM images of C19Q and HP1a in EpiGo-Control and EpiGo-KRAB cell lines. C19Q (green) was labeled by GFP and HP1a (magenta) was labeled by HaloTag. The colocalization between C19Q and HP1a was shown in merged images. c Percentage of $\mathrm{C} 19 \mathrm{Q}$ foci clusters in EpiGo-Control (dCas9) and EpiGo-KRAB (dCas9-KRAB) cell lines. C19Q cluster was defined as that all the $\mathrm{C} 19 \mathrm{Q}$ loci were confined within $1 \mu \mathrm{m}$ in each dimension. $n=200$ transfected cells in each experiment and the error bar is standard deviation (SD) from three independent experiments. d RT-qPCR analysis of SETDB1 knockdown efficiency. RNA was extracted from the EpiGo-KRAB cells lines transfected with either control siRNA or SETDB1 siRNA. $n=200$ transfected cells in each experiment and the error bar is standard deviation (SD) from three independent experiments. e 3D-SIM images of C19Q (green) and HP1a (magenta) in EpiGo-KRAB cell lines in the presence of control siRNA or SETDB1 siRNA. $\mathbf{f}$ Percentage of $\mathrm{C} 19 \mathrm{Q}$ foci clusters in the U2OS-dCas9-KRAB cells lines transfected with either control siRNA or SETDB1 siRNA. $n=200$ transfected cells in each experiment and the error bar is standard deviation (SD) from three independent experiments

mediated genomic clustering, SETDB1 was knocked down by siRNAs in EpiGo-KRAB (dCas9-KRAB) cell lines (Fig. 2d). The mRNA levels of SETDB1 decrease to $26 \%$ of the mock cells when transfected with SETDB1 siRNA. The percentage of C19Q clustering is $87 \%$ in cells transfected with control siRNA, but markedly decreased to $14 \%$ in cells transfected with SETDB1 siRNA (Fig. 2f). Finally, C19Q loci were highly dynamic in EpiGo-Control cells (Fig. 3a-c) but the mobility of C19Q loci dramatically decreased in EpiGo-KRAB cell lines (Fig. 3d-f). These results support a role of H3K9me3 in mediating phase separation of HP1 $\alpha$ droplets, large-scale genome organization, and chromatin dynamics. 
A

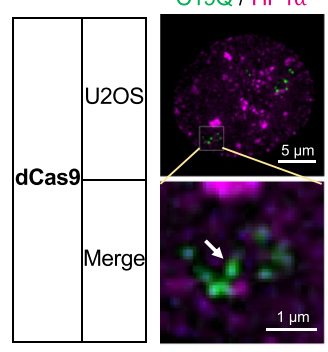

C

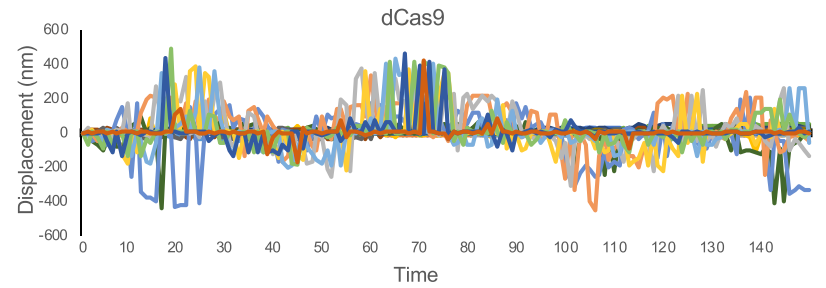

D

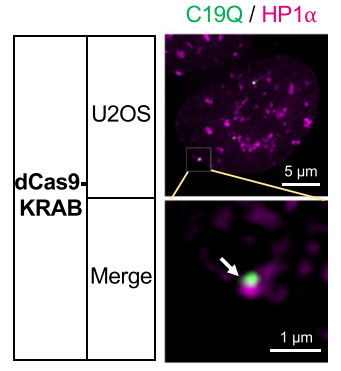

$\mathbf{F}$

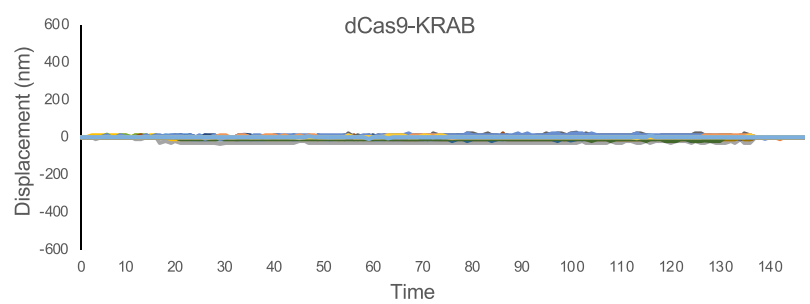

B

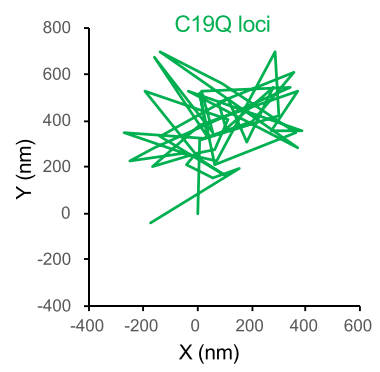

E

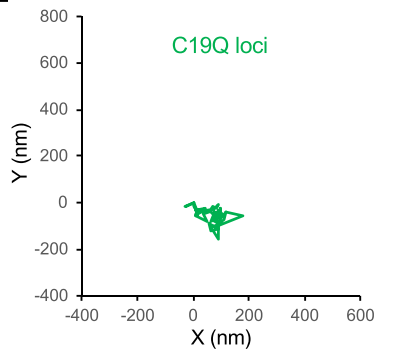

Fig. 3 Tracking the Dynamics of C19Q loci in EpiGo-KRAB cells. a Live cell tracking of C19Q loci movement in EpiGo-Control cell lines. The dynamics of C19Q (green) and HP1 a (magenta) were tracked for $30 \mathrm{~s}$. The movement of locus C19Q loci (arrowed) were recorded at $100 \mathrm{~ms}$ per frame for $30 \mathrm{~s}$. b All trajectories were shifted to start from the origin $(0,0)$ for easy comparison of the movement vectors. The locus movements were corrected for the possible movements of microscope stage. c Displacement of C19Q loci in EpiGoControl cell lines. Distance to the start point of each time point was measured and plotted. $n=20$ cells. $\mathbf{d}$ Live cell tracking of C19Q loci movement in EpiGo-KRAB cell lines. The image processing details are the same as described in a. e All trajectories were shifted to start from the origin $(0,0)$ for easy comparison of the movement vectors. The locus movements were corrected for the possible movements of microscope stage. $\mathbf{f}$ Displacement distance of C19Q loci in EpiGo-KRAB cell lines. Distance to the start point of each time point was measured and plotted. $n=17$ cells

Immunofluorescence showed extensive overlapping between H3K9me3 and HP1 $\alpha$ by conventional widefiled microscopy (Fig. 4a, b), while structured illumination microscopy (3D-SIM) showed that H3K9me3 localized nearby HP1 $\alpha$ condensates with less degree of overlapping (Fig. 4c, d). Statistic analysis showed H3K9me3 and HP1 $\alpha$ areas showed $\sim 52 \%$ overlap in images from widefield microscopy, which decrease to $25 \%$ when measured by 3D-SIM (Fig. 4e). Together with the finding that EpiGo-KRAB of C19Q loci adjacent to HP1 $\alpha$ condensates, our data suggest that the role of chromatin 
A
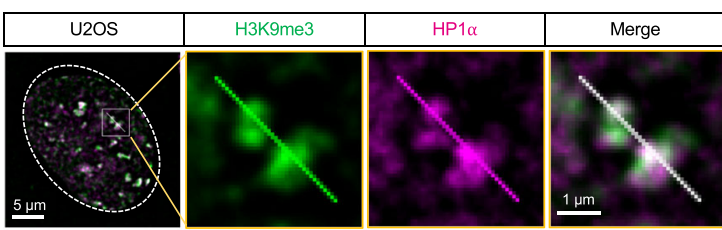

B

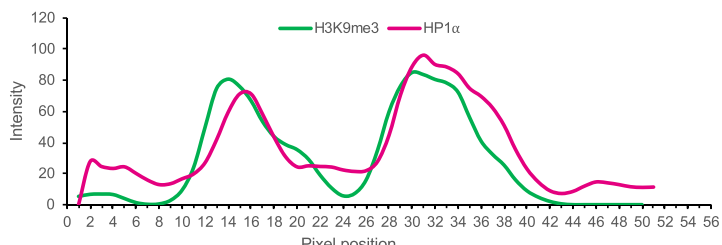

C

Pixel position
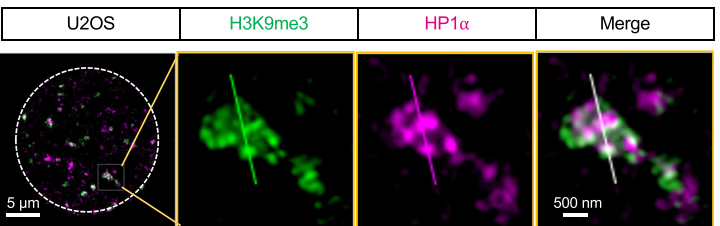

D

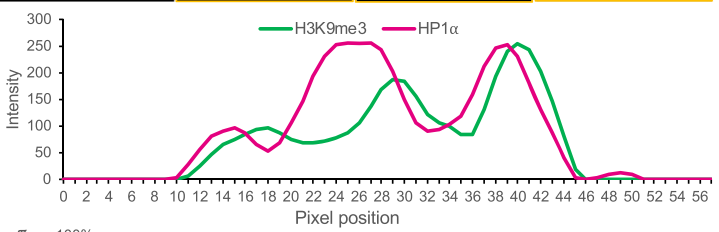

$\mathbf{E}$

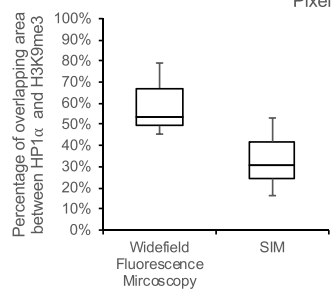

Fig. 4 Colocalization of H3K9me3 and HP1a in U2OS cells. a Imaging of H3K9me3 and HP1a colocalization by widefield fluorescence microscopy. H3K9me3 puncta (green) were detected by H3K9me3 antibody and HP1 a (magenta) was tagged by HaloTag. $\mathbf{b}$ Linescan of the vertical line. Linescan shows, from top to bottom, the intensities (arbitrary units) of $\mathrm{H3} \mathrm{K} 9 \mathrm{me} 3$ (green) and HP1a (magenta) along the line indicated in a. c Imaging of H3K9me3 and HP1a colocalization by widefield fluorescence microscopy 3D-SIM. The image processing details are the same as described in $\mathbf{a}$. $\mathbf{d}$ Linescan of the vertical line. The image processing details are the same as described in $\mathbf{b}$. e Percentage of overlapping area between HP1a and H3K9me3 from images captured by widefield fluorescence microscopy and 3D-SIM. The outer edges of the box are the 25 th and 75 th percentiles. The whiskers extend to the minimum and maximum values. $n=20$ cells in each experiment

DNA is more likely to be the anchors for facilitating HP1 $\alpha$ phase separation, rather than the composition of $\mathrm{HP} 1 \alpha$ condensates.

\section{EpiGo-KRAB-mediated H3K9me3 and genomic clustering does not result in widespread gene silencing}

We then sought to investigate the effect of ectopic H3K9me3 on transcription. The C19Q repeats are present in gene body (753 copies, 89\%), promoter or distal regions (98 copies, 11\%), and intergenic regions (158 copies, 18\%) (Fig. 5a). The C19Q-1 region spans $3.5 \mathrm{Mb}$ and contains 146 genes (Fig. 5b). This region contains clearly two sub-regions: regions 1 and 2 (Fig. 5b, c). Most genes (64\%) in region 1 are active or modestly active, while the majority of genes (79\%) in region 2 are inactive in EpiGo-Control (dCas9) cells. The peaks 


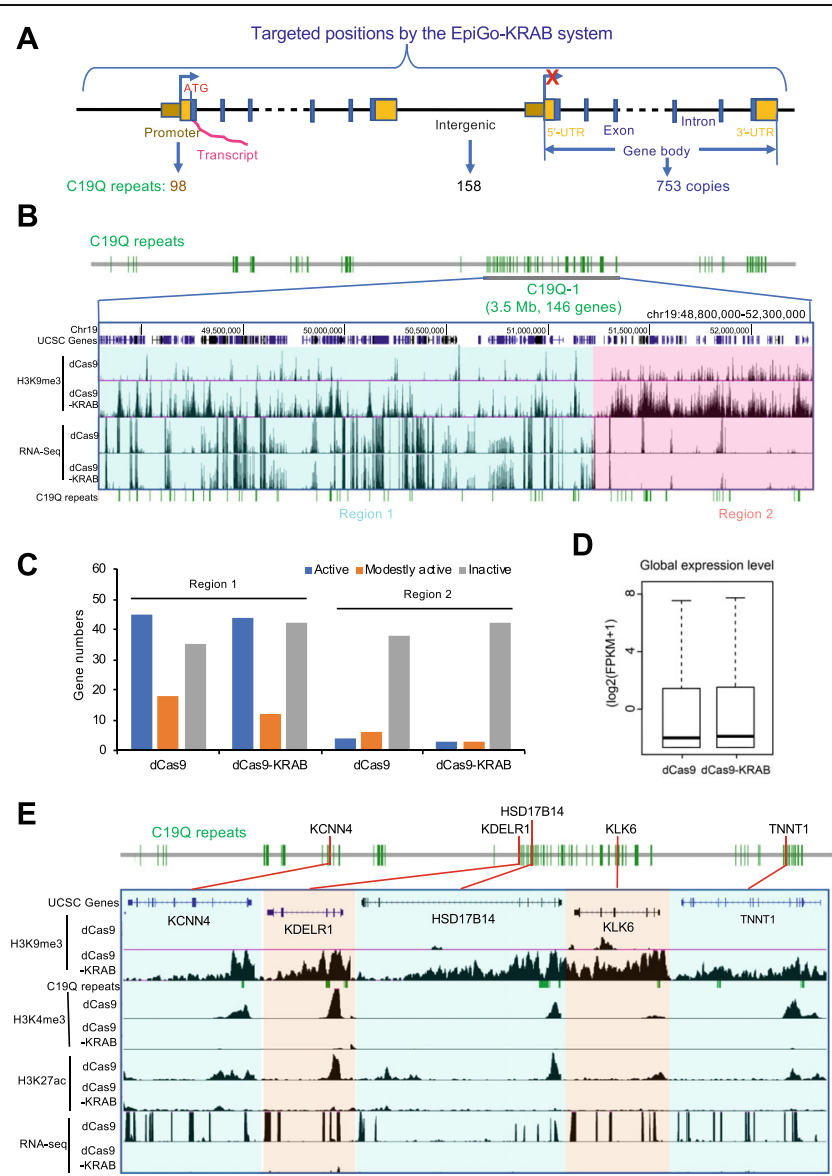

Fig. 5 The influence of H3K9me3 on transcription. a Diagram of targeted positions by the EpiGo-KRAB system, which includes promoter, gene body (5'-UTR, exon, intron, 3'-UTR), and intergenic region. There are 98 copies of C19Q repeats on promoters, 158 copies on intergenic regions, and 753 copies on gene bodies. b RNA levels in EpiGo-Control (dCas9) and EpiGo-KRAB (dCas9-KRAB) cell lines. RNA-seq was performed in these two cell lines. Region 1 (chr19: 48,800,000-51,200,000) or region 2 (chr19: 51,200,001-52,300,000) of C19Q-1 with different transcriptional states was highlighted with cyan or pink respectively. $\mathbf{c}$ Histogram shown active, modestly active, and inactive genes in region 1 and region 2 in EpiGo-Control (dCas9) and EpiGo-KRAB (dCas9KRAB) cell lines. Active genes were defined by applying a threshold of FPKM $\geq 5$, modestly active genes were applied a threshold of $1<$ FPKM $<5$, and inactive genes were used a threshold of FPKM $\leq 1$. d Global expression level in EpiGo-Control (dCas9) and EpiGo-KRAB (dCas9-KRAB) cell lines. e ChIP-seq of H3K9me3, H3K4me3, H3K27ac, and RNA-seq were performed in EpiGo-Control (dCas9) and EpiGo-KRAB (dCas9-KRAB) cell lines. KCNN4, HSD17B14, TNNT1 genes and KDELR1, KLK6 genes were highlighted with light orange or cyan respectively. All genes were marked location in $\mathrm{C} 19 \mathrm{Q}$ repeats with red lines

of EpiGo-induced ectopic H3K9me3 were negatively correlated with active transcription (Fig. 5b), as also observed for endogenous H3K9me3 genome wide (Fig. S2). We found that the expression of the majority (61 out of 73) of active genes in regions 1 and 2 was not affected by EpiGo-KRAB (Fig. 5b, c). As shown in Fig. 5d, EpiGo-KRAB does not affect the global expression levels neither. A close examination of EpiGo-KRAB targeted genes revealed that a small set of active genes are indeed silenced. For these genes, such as the top five silenced genes (KCNN4, KDELR1, HSD17B14, KLK6, and TNNT1), ectopic H3K9me3 primarily occurred at the promoter regions, which is accompanied by the loss of H3K4me3 and H3K27ac (Fig. 5e). We examined the relationship between the distance of CRISPR targets to gene promoters and their repression efficacy (Fig. S3). The result suggested that when closer the dCas 9 target is to its nearby promoter, the more likely the 
respective gene would be repressed. Such observation is also confirmed by the analyses of promoter H3K4me3 and H3K27ac changes (Fig. S3). By contrast, the orientation does not have apparent effects on gene repression. These data together suggest that EpiGo-KRABmediated global chromatin clustering does not result in widespread gene silencing beyond its direct promoter targets.

\section{Ectopic H3K9me3 spreads coutinously in inactive regions but restrictedly from transcriptional initiation sites in active regions}

To reciprocally investigate the effect of transcriptional activity on H3K9me3 spreading, we chose two areas in region 1 and region 2. KDELR1 and CYTH2 located within a $150 \mathrm{~kb}$ region in region 1. KDELR1 acquired H3K9me3 in the promoter region, which was concurrently loss of H3K4me3 and H3K27ac, and lead to gene silencing (Fig. 6a, b, $\mathrm{d}-\mathrm{f})$. CYTH2, which acquired ectopic H3K9me3 in the 3'-UTR region, did not show significant changes of promoter chromatin states and transcriptional level (Fig. 6a, b,
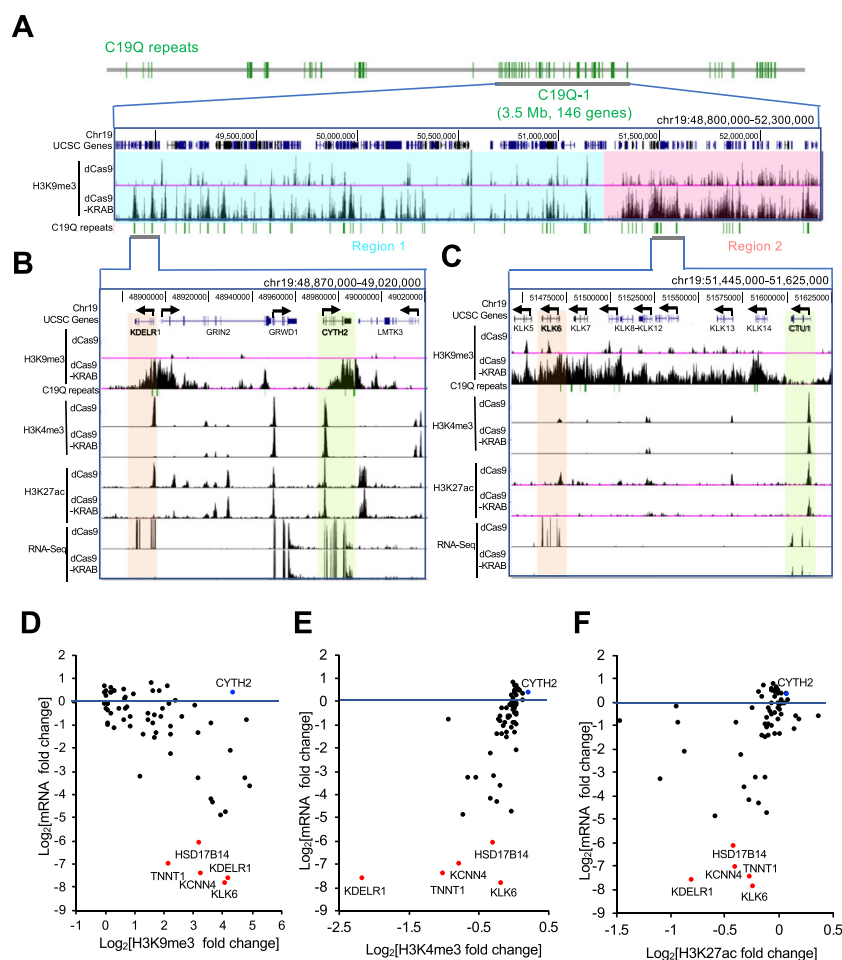

Fig. 6 EpiGo-KRAB-mediated H3K9me3 deposit does not result in widespread gene silencing. a H3K9me3 and RNA levels in EpiGo-Control (dCas9) and EpiGo-KRAB (dCas9-KRAB) cell lines. ChIP-seq of H3K9me3 and RNA-seq were performed in these two cell lines. Region 1 (chr19: 48,800,000-51,200,000, cyan) or region 2 (chr19: 51,200,001-52,300,000, pink) of C19Q-1 with different transcriptional states was highlighted with cyan or pink respectively. b H3K9me3, H3K4me3, H3K27ac states and RNA level in region 1. ChIP-seq of H3K9me3, H3K4me3, H3K27ac, and RNA-seq were performed in EpiGo-Control (dCas9) and EpiGo-KRAB (dCas9-KRAB) cell lines. KDELR1 gene or CYTH2 gene were highlighted with light orange or light green respectively. c H3K9me3, H3K4me3, H3K27ac states and RNA levelin region 2. KLK6 gene or CTU1 gene were highlighted with light orange or light green respectively. $\mathbf{d}$ Fold changes of mRNA level and H3K9me3 in the EpiGo-KRAB targeted genes. The active genes (FPKM $\geq 1)$ targeted by EpiGo-KRAB were chosen for the analysis. The five genes (KCNN4, KDELR1, HSD17B14, KLK6, and TNNT1) with most reduction in mRNA levels were marked in red. e Fold changes of mRNA level and H3K4me3 in the EpiGo-KRAB targeted genes. $\mathbf{f}$ Fold changes of mRNA level and H3K27ac in the EpiGo-KRAB targeted genes 
d-f). On the contrary, H3K9me3 spreading halted at the site of CYTH2 promoter (Fig. 6b, d). Interestingly, ectopic H3K9me3 appears to frequently exist as continuous domains in region 2 (Fig. 6a, c). For example, H3K9me3 spreads beyond C19Q repeats for hundreds of kilobases in region 2 until the proximity of an active gene CTU1 (Fig. 6c). By contrast, ectopic H3K9me3 in active region (region 1 in Fig. 6b) is intermittent and its spreading is frequently restricted, often stopping at the sites of active genes. These results raise a possibility that H3K9me3 spreads more efficiently in transcriptionally silenced regions but may be antagonized by active chromatin states.

\section{EpiGo-KRAB induces de novo heterochromatin-like domain formation}

To further examine genome organization of the active or silenced regions upon H3K9me3, Oligopaint FISH was used to visualize region 1, region 2, and region 3 (Fig. 7a and Table S2-S3). Region 1 was transcriptionally active and showed intermittent H3K9me3 in EpiGo-KRAB cell lines. Region 2 was silenced and showed more continuous H3K9me3 in EpiGo-KRAB cell lines. We also included a neighbor region 3' downstream of region 2 as a comparison (region 3). Region 3 showed strong and continuous H3K9me3 in both EpiGo-Control and EpiGo-KRAB cell lines, consistent with the fact that it is not targeted by EpiGo-KRAB. In EpiGoControl cells, region 1 showed multiple discrete foci, which partially overlap with C19Q probe but with few loci associate with HP1 $\alpha$ (Fig. 7b-d). By contrast, region 2 exists as only one focal spot in the majority of cells. It overlaps with C19Q but, surprisingly, barely associated with HP1 $\alpha$ (Fig. 7b-d), suggesting that region 2 is compacted independent of HP1 $\alpha$ association before EpiGo-KRAB induction and ectopic H3K9me3 acquisition. Region 3, which is extensively modified by H3K9me3 in EpiGo-Control cells, has one focal spot and is associated with HP1 $\alpha$ (Fig. $7 b-d$ ). These results suggested that silenced regions (region 2 or 3 ) are more compacted than active regions (region 1), and their association with $\mathrm{HP} 1 \alpha$ appears to be related to H3K9me3 strength. Indeed, in EpiGo-KRAB cells, region 1 collapsed into one focal spot and associated with HP1 $\alpha$ (Fig. 7e-g). Region 2 again had only one focal spot, but now became highly associated with HP1 $\alpha$ (Fig. 7e-g). These results indicated that EpiGo-KRAB promotes its association with HP1 $\alpha$ condensates and further chromatin compaction perhaps in an H3K9me3 density-dependent manner.

\section{EpiGo-KRAB-induced large-scale rearrangement of chromatin compartmentalization}

Genome is organized in 3D from sub-kilobase to megabase scales and segregated into domains in cellular nucleus [4]. Genome compartmentalization occurs at the hundreds of kilobases to megabase scales from Hi-C heatmap [11]. To understand how epigenetic manipulation affects compartmentalization in active and silenced regions, $\mathrm{Hi}-\mathrm{C}$ was performed on U2OS-EpiGo-Control (dCas9) and U2OS-EpiGo-KRAB (dCas9-KRAB) cell lines. As a control, no drastic changes of global compartmentalization were observed in the $\mathrm{Hi}-\mathrm{C}$ matrix of the genome upon EpiGo-KRAB induction (data not shown). Focused on the $\mathrm{C} 19 \mathrm{Q}$ region, which showed substantial changes of H3K9me3 states, we found drastic rearrangement of local compartments (Fig. 8a, b). For instance, the entire region 2 and region 3 fused to become a large compartment in EpiGo-KRAB cells, which even erodes part of region 1 (1/2/3 compartment) (Fig. 8b). Throughout the regions, we have 
A

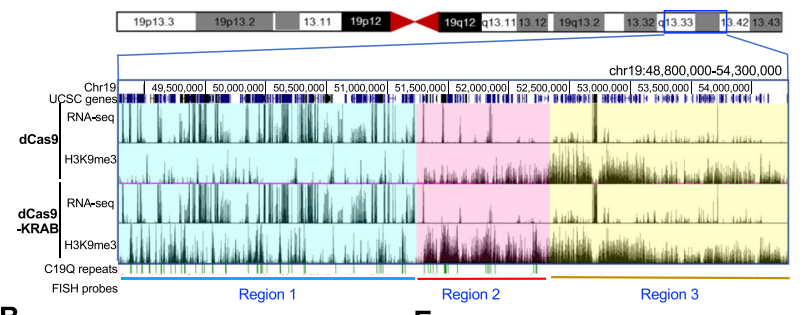

B

E

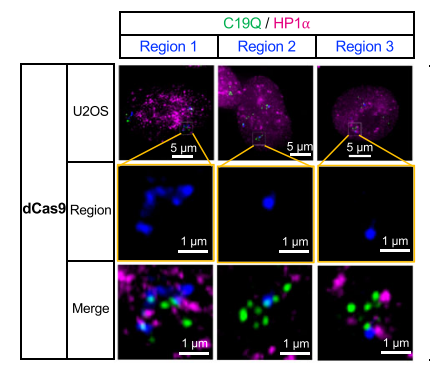

\begin{tabular}{|l|l|l|}
\hline \multicolumn{3}{|c|}{ C19Q/HP1 $\alpha$} \\
\hline Region 1 & Region 2 & Region 3 \\
\hline
\end{tabular}

C
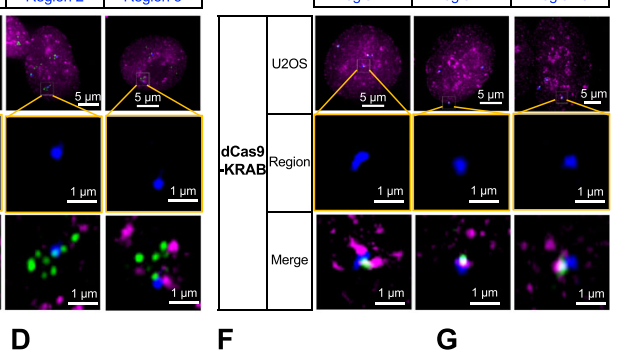

$\mathbf{F}$

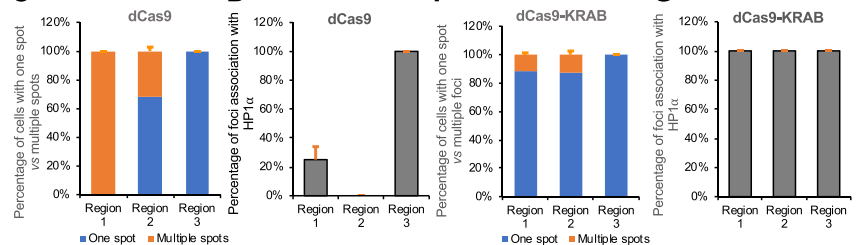

Fig. 7 EpiGo-KRAB induced de novo heterochromatin-like domain formation. a H3K9me3 states and RNA levels of region 1, region 2, and region 3 in EpiGo-Control (dCas9) and EpiGo-KRAB (dCas9-KRAB) cell lines. Region 1 (chr19: 48,800,000-51,200,000) representing an active region, region 2 (chr19: 51,200,001-52,300,000) representing a silenced region, and region 3 (chr19: 52,300,001-54,300,000) containing endogenous H3K9me3 was highlighted in cyan, pink, and yellow respectively. The target sites of C19Q repeats were shown in green. Oligopaint FISH probes were designed to detect region 1, region 2, and region 3 respectively. $\mathbf{b}$ Images of C19Q, HP1 a and region 1, 2, or 3 in EpiGo-Control cell lines. C19Q (green) was labeled by GFP using CRISPR imaging, HP1a (magenta) was labeled by HaloTag, and region 1, 2, or 3 (blue) was labeled by in situ hybridization with DNA probes conjugated to the dye Alexa-647. The colocalization among C19Q, HP1a, and region 1, 2 or, 3 was shown in merged images. c Percentage of cells with one spot vs multiple spots in region 1, 2, or 3 of EpiGo-Control (dCas9) cell lines. $n=200$ transfected cells in each experiment and the error bar is standard deviation (SD) from three independent experiments. $\mathbf{d}$ Percentage of foci association with HP1a in EpiGo-Control cell lines. The association or nonassociation is defined by overlapping (regardless of the degrees) or non-overlapping between foci and HP1a condensates. $n=16,16$, and 12 cells from left to right respectively. e Images of C19Q, HP1a and region 1, 2, or 3 in EpiGo-KRAB cell lines. All the conditions are the same as $\mathbf{b}$ except the dCas9 was replaced by dCas9-KRAB. $\mathbf{f}$ Percentage of cells with one spot vs multiple spots in region 1,2, or 3 of EpiGo-KRAB (dCas9-KRAB) cell lines. $n=$ 200 transfected cells in each experiment and the error bar is standard deviation (SD) from three independent experiments. $\mathbf{g}$ Percentage of foci association with HP1a in EpiGo-KRAB cell lines. The association or nonassociation is defined by overlapping (regardless of the degrees) or non-overlapping between foci and HP1a condensates. $n=10,15$, and 12 cells from left to right respectively

found a number of regions with $\mathrm{C} 19 \mathrm{Q}$ repeats that show increased interactions with their neighbor regions, leading to compartment merging (red arrows, Fig. 8a). These results indicated that EpiGo-KRAB-induced extensive re-arrangement of chromatin compartments. We reasoned that this is possibly because $\mathrm{H} 3 \mathrm{~K} 9 \mathrm{me} 3$ marked regions anchor chromatin to HP1 $\alpha$ condensates, which breaks existing compartmentalization [28] and form new compartments. Therefore, the final compartments in the genome may be decided by the overall topology executed through a default compartmentalization (gene density and transcriptional state-correlated) altered by architectural proteins and epigenetic states. 

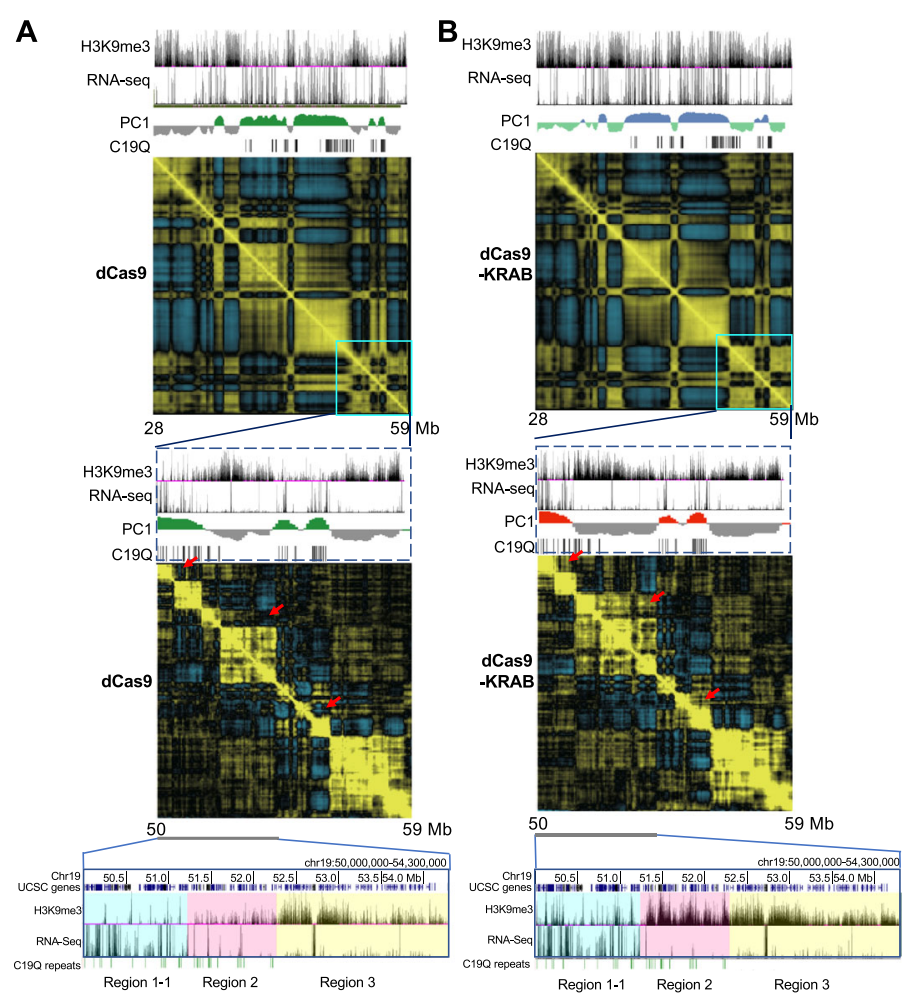

Fig. 8 EpiGo-KRAB induced rearrangement of chromatin compartmentalization. a Hi-C heatmap shown the compartmentalization of the C19Q region in EpiGo-Control (dCas9) cells. H3K9me3 state, RNA level, PC1, and C19Q repeats (target sites) shown at the top of the Hi-C heatmap (Chr19: 28,000,000-59,000,000). Hi-C heatmap of a local region (chr19: 50,000,000-59,000,000) was used to show the local compartmentalization. Region 1-1 (a partial region 1), region 2, and region 3 were shown in cyan, pink, and yellow respectively. $\mathbf{b}$ Hi-C heatmap shown the compartmentalization of the C19Q region in EpiGo-KRAB (dCas9) cells. All the data processing and display are the same as a

\section{The compartment A-to-B switch induced by EpiGo-KRAB is associated with multiple} factors

As EPIGO-KRAB would induce compartment re-organization, we first asked what would be the threshold of dcas9-KRAB target density to achieve a compartment A-to-B switch. A close examination revealed that out of 49 compartment A regions (100 kb bin) with target sequences in dCas9 cells, surprisingly only 4 of them were converted to compartment $B$ in dCas9-KRAB cells (A-to-B), while the rest 45 remained as compartment A (A-to-A), raising the possibility that the target density may need to exeed a threshold to flip a compartment A to B. Indeed, we found that the average target densities (per $100 \mathrm{~kb}$ bin) in A-to-B regions $(n=10,49,18,20$, respectively, see red box in Fig. 9a, b) are much higher than A-to-A regions (Fig. 9c), supporting the notion that high target density is needed to achieve chromatin organization alterations.

Nevertheless, the dense targeting of dCas9-KRAB is clearly not a simple rule, as some A-to-A regions and A-to-B regions show high target density (Fig. 9c). One A-to-A example can be seen in Fig. 9a, b which show dense presence of dCas9-KRAB targets (green box). Notably, these regions appear to enrich for active genes (Fig. 9a, b), raising the possibility that additional factors may be also involved in compartment switch. Notably, our recent study also showed that during SCNT, many compartments A and B remain constant between mouse donor cells and SCNT embryos, and they preferentially 
A

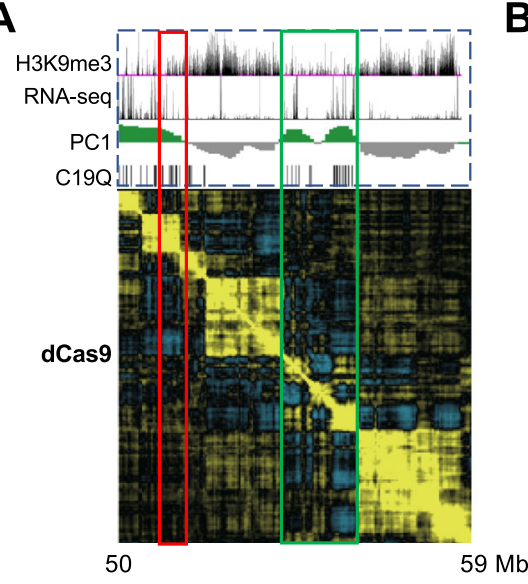

C compartment dynamics

vs. target density

dCas9 vs dCas9-KRAB

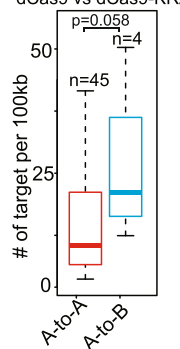

D Compartment dynamics vs. gene density

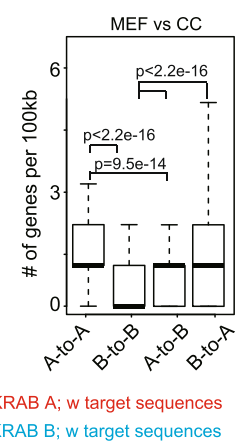

B

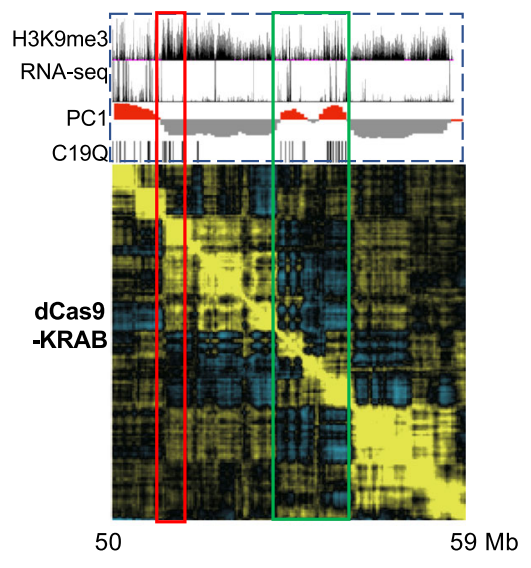

E Compartment dynamics

vs. gene density

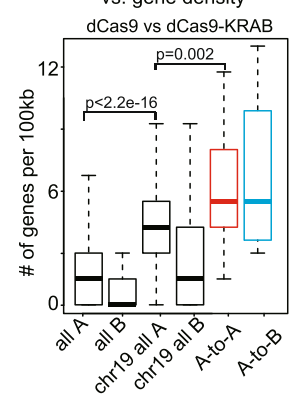

$\square$ dCas9 A-to KRAB A; w target sequences
$\square$ dCas9 A-to KRAB B; w target sequences

$\mathbf{F}$
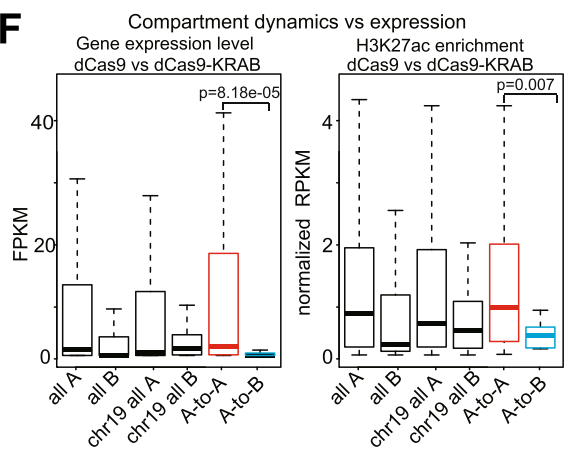

G Compartment dynamics

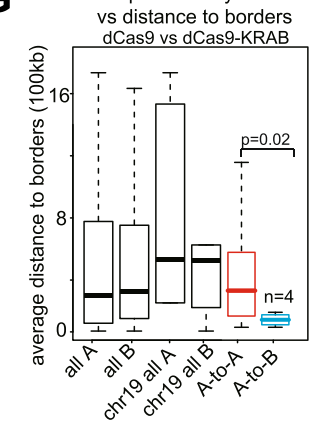

Fig. 9 (See legend on next page.) 
(See figure on previous page.)

Fig. 9 Compartment switch is regulated by multi-factors. a Hi-C heatmap showing the compartmentalization of the C19Q region in EpiGo-Control (dCas9) cells (chr19: 40,000,000-50,000,000). H3K9me3, RNA-seq, and PC1 tracks are also shown. Red box, A-to-B switched regions upon dCas9-KRAB induction. Green box, A-to-A regions upon dCas9KRAB induction. $\mathbf{b} \mathrm{Hi}-\mathrm{C}$ heatmap showing the compartmentalization of the $\mathrm{C} 19 \mathrm{Q}$ region in EpiGo-KRAB cells. All the data processing and display are the same as a. c Boxplots showing the dCas9-KRAB target sequence density for Ato-A and A-to-B regions in dCas9 and dCas9-KRAB cells. Only compartment A regions with target sequences were selected for analysis. P values calculated by Wilcoxon rank-sum test (two-sided) are also shown. $\mathbf{d}$ Left, boxplots showing the gene density in each class for mouse cell lines (MEF A-to-CC B, MEF B-to-CC A, MEF A-to-CC B, MEF Bto-CC A). Right, boxplots showing the gene density in each class for human cell lines (hES A-to-TB B, hES B-to-TB A, hES A-to-TB B, hES B-to-TB A). MEF, mouse embryonic fibroblast; CC, cumulus cells; hES, human embryonic stem cells; TB, trophectoderm. Hi-C data were obtained from GSE52457 and GSE139430. P values calculated by Wilcoxon rank-sum test (two-sided) are also shown. e Boxplots showing the gene density in each class. Whole genome compartment A/B and all chr19 compartment AVB in dCas9 cells are similarly analyzed and shown as control. P values calculated by Wilcoxon rank-sum test (two-sided) are also shown. $\mathbf{f}$ Left, boxplots showing the gene expression level in each class. Right, boxplots showing the H3K27ac enrichment at promoters for genes located in each class. Whole genome compartment A/B and all chr19 compartment AVB in dCas 9 cells were are similarly analyzed and shown as controls. P values calculated by Wilcoxon rank-sum test (two-sided) are also shown. $\mathbf{g}$ Boxplots showing the average distance towards $A / B$ borders in each class. Whole genome compartments $A / B$ and all chr19 compartments A/B in dCas9 cells were used as control. All A-to-B regions in chr19q arm with dCas9-KRAB targets were similarly analyzed and shown. P values calculated by Wilcoxon rank-sum test (two-sided) are also shown

have the highest and lowest gene densities respectively [29]. By contrast, switched compartment regions tend to have intermediate gene density [29]. We now confirmed such results in additional mouse cells (MEF vs. cumulus cells [29, 30]) and human cells (hESC vs trophectoderm [31]) (Fig. 9d). Similar results were also reported for laminaassociated domains (LADs) which are closely associated with compartments A/B [32]. Regions with high and low gene density tend to be constant LADs (cLADs) and constant inter-LADs, while variable LADs (vLADs) preferentially show intermediate gene density and frequently reside at the boundary of LADs and iLADs [33]. Our analyses revealed that compared to the whole genome, chr19 has unusually high levels of gene density, including both A-to-B and A-to-A regions (Fig. 9e), suggesting that these regions may be naturally resistant to A-to- $B$ switches. In addition, A-to-A regions show higher gene expression and histone acetylation levels than A-to-B regions, supporting that A-to-A regions are more likely to be stable compartment A (Fig. 9f). Finally, it was reported that variable LADs (vLADs) frequently reside at the boundary of LADs and iLADs [33]. Similarly, we found that A-to-B regions are preferentially located near A/B boundaries (Fig. 9g, see example in Fig. 9a, b, red box).

In sum, we show that compartment switch is likely regulated by multiple factors including dcas9-KRAB binding, closeness to A-B borders, and gene density. Compartment A with targeted sequences in C19Q show particularly high gene density in most regions (Fig. 9e), suggesting that these regions may be naturally more resistant to A-to-B switch, unless they have abundant sgRNA targets and/or are near compartment A-B boundaries.

\section{Discussion}

Despite the long-observed correlation, whether epigenetic modifications can regulate 3D genome architecture in live cells remains poorly understood. The EpiGo-KRAB system provides a powerful tool to allow $\mathrm{H} 3 \mathrm{~K} 9 \mathrm{me} 3$ at specific genes or regions and track their changes of location, structure, and dynamics. Our data show that EpiGo-KRAB is able to mediate de novo heterochromatin-like domain formation in a dynamic and 
stepwise process (Fig. 10): (1) EpiGo-KRAB induces ectopic H3K9me3 at defined loci which further spread depending on the existing chromatin states and transcriptional activities, (2) EpiGo-KRAB-mediated H3K9me3 promotes chromatin anchoring to HP1 $\alpha$ condensates, and (3) EpiGo-KRAB-mediated heterochromatin-like domain, possibly by phase separation. These results also suggest neither non-promoter ectopic H3K9me3 per se nor H3K9me3-mediated chromatin compaction and phase separation with HP1 $\alpha$ condensates is sufficient for gene silencing. In fact, active transcription can persist in EpiGo-KRAB-induced cells and appear to be able to antagonize H3K9me3 spreading. It would be worthy to further explore how transcription and H3K9me3 interplay in space and time with diverse biological context.

Another interesting question is what degree of $\mathrm{H} 3 \mathrm{~K} 9 \mathrm{me} 3$ is required for HP1 $\alpha$ condensate association, chromatin local compaction, or genome compartmentalization. Here, by analyzing different regions with distinct transcriptional activities, we found that repressed regions can form compact DNA without associating with HP1 $\alpha$ condensate, which is consistent to recent finding that compaction of mouse heterochromatin foci can be independent of HP1 [34]. However, high levels of ectopic H3K9me3 may be necessary to promote $\mathrm{HP} 1 \alpha$ association and chromatin compaction in active regions.

Finally, drastic changes of epigenetic modifications such as H3K9me3, H3K27me3, and DNA methylation occur in stem cell differentiation, embryonic development, and many diseases [35-37]. With the EpiGo system, it will be interesting to explore how tissue-specific epigenetic modifications are established and maintained at kilobase to megabase scales and how they regulate chromatin architecture, gene expression, and cell fate decision.

\section{EpiGo-KRAB mediated de novo heterochromatin-like domain formation}
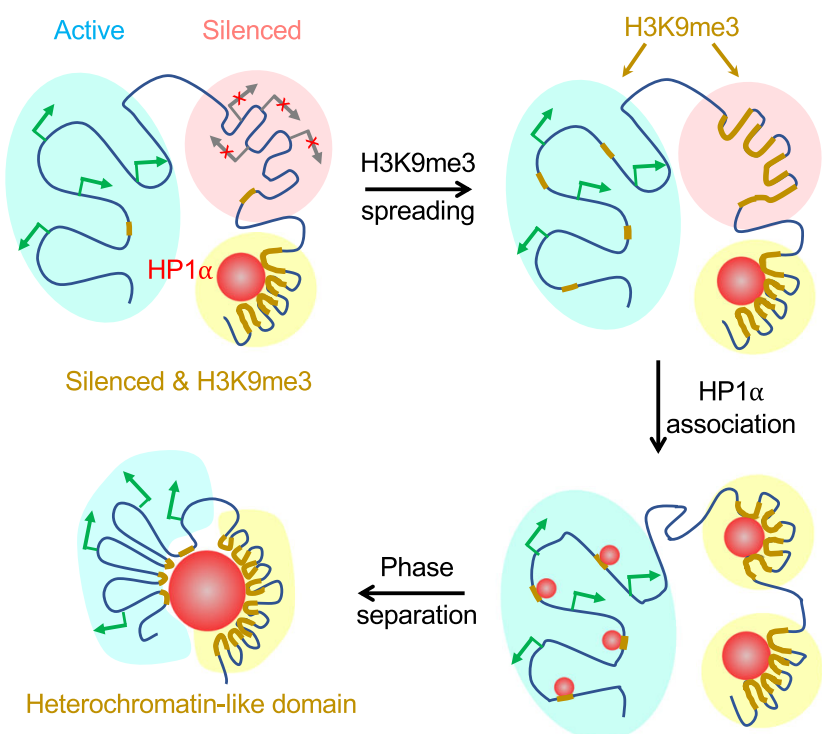

Fig. 10 A model for genome organization mediated by H3K9me3 in living cells. H3K9me3 spread continuously over hundreds of kilobases to megabases in silenced regions, but the spreading frequently stops at the transcriptional initiation sites in active regions. Loci or regions with H3K9me3 decorate on the surface of HP1a condensates, which mediates chromatin local compaction. H3K9me3 and local compaction does not result in widespread gene silencing in active regions, but induces large scale chromatin compartmentalization possibly by phase separation 


\section{Methods}

\section{Cell culture}

The U2OS (human bone osteosarcoma epithelial, female) cell and HEK293T cells (human embryonic kidney epithelial, female) were cultured in DMEM (Life Technologies) with high glucose in 10\% FBS (fetal bovine serum, Life Technologies). All cells were cultured at $37^{\circ} \mathrm{C}$ and $5 \% \mathrm{CO}_{2}$ in a humidified incubator.

\section{Chromosome-specific repeats for the EpiGo system}

Mining of chromosome-specific repeats was described previously with some modifications [38]. The human reference genome (assembly GRC h37/hg19) was downloaded from the UCSC Genome Browser (http://genome.ucsc.edu) to find target regions and design guide RNAs. The bioinformatics tool Jellyfish [39] was used to find all 15-mers (12-mers ending with NGG or starting with $\mathrm{CCN}$ ) with at least 5 copies in any chromosome. The 15-mers with more than 100,000 targets were filtered out. Each candidate 15-mer was searched for off-targets in all other chromosomes. The candidate 15-mer was discarded if there was any cluster of 5 offtargets or more within any $50 \mathrm{~kb}$ region. The C19Q repeats (Table S1), which consists of 836 copies of CRISPR target sites (TCCCTCAGACCCnGG) spanning 17 megabases at the q-arm of chromosome 19, was chosen as EpiGo targets in this study. The overlapping genes including the intron/exon information were extracted from the GENCODE Genes track (version 27lift37), downloaded from the UCSC Genome Browser.

\section{Plasmids construction}

The expression plasmid pHAGE-TO-dCas9 has been described previously [17, 21], in which HSA-P2A was inserted at the N-terminal of dCas9 resulting in pHAGETO-HSA-P2A-dCas9 and KRAB was then subcloned into the C-terminal of dCas9 resulting pHAGE-TO-HSA-P2A-dCas9-KRAB. The expression plasmid pHAGEEFS-PCP-GFP has been described previously [21]. The expression vector for guide RNA was based on the pLKO.1 lentiviral expression system, in which TetR-BFPP2A-2XPP7 was inserted right after the phosphoglycerate kinase (PGK) promoter, resulting pTetR-P2A-BFP-2XPP7. The expression plasmid for guide RNAs targeting to $\mathrm{C} 19 \mathrm{Q}$ was made by using the rapid guide RNA construction protocol described previously [38].

The donor plasmid for knock-in of HaloTag at the C-terminal of HP1 $\alpha$ was made by Golden Gate cloning. The donor consists of three fragments: a 400-bp fragment just upstream of stop codon of HP1 $\alpha$ (left arm), HaloTag coding sequences, and a 400-bp fragment just downstream of stop codon of HP1 $\alpha$ (right arm). Left arm and right arm fragments were amplified from U2OS genomic DNA isolated with Cell DNA isolation mini kit (Vazyme). The HP1 $\alpha$-HaloTag donor was assembled by Golden Gate cloning method into pDONOR vector [38], resulting in pDONOR-HP1 $\alpha$-HaloTag. The guide RNA targeting sequences AAACAGCAAAGAGCTAAAGG spanning the stop codon (TAA, underlined) of HP1 $\alpha$ and cloned into guide RNA expression vector pLHsgRNA2 [21], resulting in PLH-sgRNA2-HP1 $\alpha$. 


\section{Generation of U2OS-HP1a-HaloTag cell lines}

For knock-in of HaloTag into HP1 $\alpha$ locus, U2OS cells were co-transfected with $200 \mathrm{ng}$ of pHAGE-TO-Cas9, $600 \mathrm{ng}$ of pLH-sgRNA1-HP1 $\alpha$, and $600 \mathrm{ng}$ pDONOR-HP1 $\alpha-$ HaloTag using Lipofectamine 2000 (Life Technologies) for $6 \mathrm{~h}$ and then replaced culture media containing $2 \mathrm{nM}$ HaloTag-JF549. The transfected cells were cultured for additional 24-48 $\mathrm{h}$ before examining the knock-in efficiency by fluorescent imaging or flow cytometry.

Fluorescence imaging was used to check the proper localization of HP1 $\alpha$-HaloTag. The HaloTag positive cells was sorted by BD FACS Aria III equipped with $561 \mathrm{~nm}$ excitation laser, and the emission signals were detected by using filter at 610/20 nm (wavelength/bandwidth) for HaloTag-JF549. The localization of HP1 $\alpha$-HaloTag was examined again under fluorescence microscope after cultured for additional two weeks. The resulting cell line was named U2OS-HP1 $\alpha$-HaloTag.

\section{Generation of U2OS-EpiGo cell lines}

For generation of U2OS-EpiGo cell lines, lentivirus for PCP-GFP, sgRNA, dCas9, or dCas9-KRAB was used. HEK293T cells were seeded into 6-well and transfected with $0.5 \mu \mathrm{g}$ pCMV-dR8.2-dvpr, $0.3 \mu \mathrm{g}$ pCMV-VSV-G, and $1.5 \mu \mathrm{g}$ of transfer plasmid (pHAGE-EFS-PCP-GFP, pTetR-P2A-BFP-sgRNA1-2XPP7-C19Q, pHAGE-TO-dCas9, or pHAGE-TO-dCas9-KRAB) using Lipofectamine 2000 (Invitrogen) following manufacturer's protocol. After $48 \mathrm{~h}$, supernatant was harvested and filtered with $0.45-\mu \mathrm{m}$ polyvinylidene fluoride Syringe filters. The filtered supernatant was titrated, and either directly used or concentrated. The concentration was performed by using Lentivirus Concentration Reagent (Biodragon-immunotech. Inc). The concentrated viral particles were immediately used for transduction or stored at $-80^{\circ} \mathrm{C}$ in aliquots.

U2OS-HP1 $\alpha$-HaloTag cells were grown to $40 \%$ confluency in 6-well plates and viral supernatants for PCP-GFP, sgRNA targeting to C19Q, dCas9, or dCas9-KRAB were added for transduction. Fresh media containing $500 \mathrm{ng} / \mathrm{ml}$ doxycyline (Sigma-Aldrich) was used $48 \mathrm{~h}$ post-infection. After additional 24h, Alexa Fluor ${ }^{\odot} 647$ anti-mouse CD24 Antibody (BioLegend) for HSA were added before cell sorting. Expression levels of PCP-GFP, sgRNA (BFP) and dCas9 (AlexaFluor 647) or dCas9-KRAB (AlexaFluor 647) was examined by BD FACS Aria III equipped $405 \mathrm{~nm}, 488 \mathrm{~nm}$, and $647 \mathrm{~nm}$ excitation lasers, and the emission signals were detected by using filters at $450 / 40 \mathrm{~nm}$ (wavelength/bandwidth) for BFP, 530/30 nm for GFP, and 662/20 nm for AlexaFluor 647. To generate single colonies, single cells with different levels of PCP-GFP, sgRNA, and dCas9 or dCas9-KRAB were sorted into 96-well plates. After 10-14 days, triple-positive colonies were further examined for CRISPR-based labeling of C19Q foci under fluorescence microscope. The C19Q labeled cells were propagated and named U2OS-EpiGoControl (PCP-GFP, C19Q sgRNA and dCas9) and U2OS-EpiGo-KRAB (PCP-GFP, C19Q sgRNA and dCas9-KRAB) respectively.

\section{Oligopaint FISH probe design and preparation}

Oligopaint FISH probes targeting the q-arm of chromosome 19 were designed according to OligoMiner (https://github.com/brianbeliveau/OligoMiner) and probe datasets for Human chromosome 19 from hg19 with mining settings of Balance (https:// 
oligopaints.hms.harvard.edu/genome-files). Here we focused on the region of chr19: 48.8-54.3 Mb (hg19) including region A (chr19: 48.8-51.2 Mb), region B (chr19: 51.2$52.3 \mathrm{Mb}$ ), and region $\mathrm{C}$ (chr19: 52.3-54.3 Mb). The detail design of region $\mathrm{A}$, region $\mathrm{B}$, and region $\mathrm{C}$ probes were shown in Table S2 and Table S3. The oligo pool for Oligopaint FISH probes was ordered from Synbio Technologies Inc. The preparation of Oligopaint FISH probes has been described previously [13, 40]. Briefly, the oligo pools for region $\mathrm{A}$, region $\mathrm{B}$, and region $\mathrm{C}$ were amplified via limited-cycle PCR (Vazyme), with forward primer and corresponding reverse readouts (one per $300 \mathrm{~kb}$ region) with $\mathrm{T} 7$ promoter sequence TAATACGACTCACTATAGGG appended to its 5' end. Each pool was purified by AxyPrep DNA Gel Extraction Kit (Axygen). The oligo pools for region A, region $\mathrm{B}$, or region $\mathrm{C}$ were used as templates for in vitro transcription by HiScribe T7 quick high yield RNA synthesis kit (NEB). The obtained RNA products were converted back to DNA oligo probes via reverse transcription (RT) with common RT primer (ATCGAC CCGGCATCAACGCCACGATCAGCT) conjugated with a 5' AlexaFluor 647 using Maxima H Minus RT enzyme (Thermo Fisher). The intermediate RNA products were removed with $\mathrm{NaOH}$-EDTA solution in $95^{\circ} \mathrm{C}$ for $10 \mathrm{~min}$, and the single-strand DNA oligo probes were purified via DNA clean \& concentrator-25 (D4033, Zymo Research). The ssDNA probes were either directly used for Oligopaint FISH or store in aliquots at $-80^{\circ} \mathrm{C}$.

\section{Immunofluorescence}

Cells were cultured on \#1 coverslip (Electron Microscopy Sciences), and then fixed with $4 \%$ PFA for $10 \mathrm{~min}$, washed three times with 1 XPBS and permeabilized by $0.1 \%$ Triton $\mathrm{X}-100$ in $1 \mathrm{XPBS}$ for $10 \mathrm{~min}$. Cells were incubated with rabbit polyclonal antibodies against H3K9me3 (1:1000; Active Motif) in the 1XPBS for $1 \mathrm{~h}$ at room temperature. After washed three times with 1 XPBS and incubated with Goat anti-rabbit lgG $(\mathrm{H}+\mathrm{L})$ secondary antibody (1:500, Invitrogen) for $1 \mathrm{~h}$. After washed three times with 1XPBS, coverslips were mounted with Prolong antifade reagents (Invitrogen) for imaging.

\section{ImmunoFISH}

$1 \times 10^{5}$ of U2OS-EpiGo cells were cultured on the \#1 coverslip (Electron Microscopy Sciences) and added $500 \mathrm{ng} / \mathrm{ml}$ doxycycline for induction of dCas9 or dCas9-KRAB expression. After $72 \mathrm{~h}$, cells were fixed with $4 \%$ PFA for $10 \mathrm{~min}$, washed by 1 XPBS for three times, and permeabilized with PBS-0.5\%Triton X-100 for $10 \mathrm{~min}$.

Cells were incubated with Rabbit anti-GFP polyclonal antibody (ab290, Abcam) in the $1 \mathrm{XPBS}$ for $1 \mathrm{~h}$ at room temperature. After washed three times with 1XPBS and incubated with Alexa Fluor 488 Goat anti-rabbit $\lg G(\mathrm{H}+\mathrm{L})$ secondary antibody (1:500, Invitrogen) for $1 \mathrm{~h}$. After washed three times with 1XPBS, cells were fixed with 4\% PFA again for 20 min. The fixed cells were treated with $0.1 \mathrm{~N} \mathrm{HCl}$ for $5 \mathrm{~min}$ and washed with 2XSSCT $(300 \mathrm{mM} \mathrm{NaCl}, 30 \mathrm{mM}$ sodium citrate, and $0.1 \%$ Tween-20), followed by incubation with $50 \%$ (vol/vol) formamide in 2XSSCT in $60^{\circ} \mathrm{C}$ for $20 \mathrm{~min}$. Samples were denatured at $78^{\circ} \mathrm{C}$ for 3 min with $20 \mu$ hybridization buffer (2XSSC, $50 \%$ formamide, $10 \%$ dextran sulfate, $0.2 \%$ Tween-20 mixed with $\sim 1.6 \mu \mathrm{M}$ total DNA oligo probes) and incubated at $42{ }^{\circ} \mathrm{C}$ for $16 \mathrm{~h}$ in a humidified chamber. Samples were washed by 2XSSCT for 4 times at $60^{\circ} \mathrm{C}$ and 2 times at room temperature. The cells were washed by 1 XPBS with $0.5 \mathrm{M} \mathrm{NaCl}$ before mounted with Prolong antifade reagents (Invitrogen) for imaging. 


\section{Live cell imaging}

All live cell imaging was carried out on a DeltaVision $\mathrm{OMX}^{\mathrm{Tm}} \mathrm{V} 4$ imaging system (GE Healthcare), equipped with a 60x (NA 1.42) Plan Apo oil-immersion objective (Olympus). The cells were cultured on No. 1.0 glass bottom dishes (MatTek). The microscope stage incubation chamber was maintained at $37^{\circ} \mathrm{C}$ and $5 \% \mathrm{CO}_{2}$. HaloTag-JF549 was excited at $561 \mathrm{~nm}$, and its emission was collected using filter at $609 / 37 \mathrm{~nm}$ (wavelength/ bandwidth); GFP was excited at $488 \mathrm{~nm}$ and collected using filter at $498 / 30 \mathrm{~nm}$ (wavelength/bandwidth). Imaging data were acquired by DeltaVision Elite imaging (GE Healthcare Inc.) software. Tracking C19Q cluster formation was performed for $4 \mathrm{~h}$ and images were captured every $30 \mathrm{~min}$. For the representative images, the raw data were deconvoluted by softWoRx (GE Healthcare Inc.) software.

\section{D-SIM procedure}

3D-SIM was performed on a DeltaVision OMX V4 system (GE Healthcare) equipped with a 60x (1.42 NA) Plan Apo oil-immersion objective (Olympus) and six laser (405, $445,488,514,568$, and $642 \mathrm{~nm}$ ). Image stacks were captured and were reconstructed using softWoRx (GE Healthcare). Images were registered with alignment parameters obtained from calibration measurements with $100 \mathrm{~nm}$ diameter TetraSpeck Microspheres with four colors (Molecular Probes).

\section{SETDB1 knockdown}

SETDB1 siRNA (GGCAAGAAGAGAACUAAGA) and Control siRNA were purchased from GenePharma. EpiGo-KRAB cell lines were transfected with control siRNA or SETDB1 siRNA by SiRNA-Mate (GenePharma) according to the manufacture's instruction. Cells were collected $48 \mathrm{~h}$ after transfection. For RT-qPCR, total RNAs were isolated using quick-RNA MiniPrep kit (Zymo Research Inc.) according to manufacturer's instruction. RNA was converted to cDNA using HifairTM II 1st Strand cDNA Synthesis SuperMix (Yeasen). Quantitative PCR was carried out on StepOnePlusTM by using Hieff UNICON ${ }^{\circ}$ Power qPCR SYBR Green Master Mix (Yeasen). All qPCR data is represented as the mean $+/$ - standard deviation of three replicates. Primer sequences for SETDB1 and GAPDH are as follows: SETDB1 forward primer (AGGAACTTCGGCAT TTCATCG) and SETDB1 reverse primer (TGTCCCGGTATTGTAGTCCCA); GAPD $\mathrm{H}$ forward primer (CGACCACTTTGTCAAGCTCA) and GAPDH reverse primer (AGGGGTCTACATGGCAACTG).

\section{ChIP-seq}

Cells were collected and crosslinked by $1 \%$ formaldehyde. Cells were suspended in cell lysis buffer B (50 mM Tris- $\mathrm{HCl} \mathrm{PH}$ 8.0, 20 mM EDTA, 0.3\% SDS, freshly added protease inhibitor) for $10 \mathrm{~min}$ on ice before sonication. One milligram beads (Invitrogen) were washed with PBS $+5 \mathrm{mg} / \mathrm{mL}$ BSA for three times and incubated with $5 \mu \mathrm{g}$ antibody at $4{ }^{\circ} \mathrm{C}$ for $6-8 \mathrm{~h}$ before adding chromatin. Sonicated chromatin was diluted in dilution buffer (16.7 mM Tris-HCl PH 8.0, 1.1\% Triton X-100, $1.2 \mathrm{mM}$ EDTA, $167 \mathrm{mM} \mathrm{NaCl}$, freshly added protease inhibitor) and was incubate with pre-treated beads and antibodies by rotation at $4{ }^{\circ} \mathrm{C}$ overnight. Then, beads were washed with washing buffer (50 $\mathrm{mM}$ Hepes PH 8.0, 1\% NP-40, 0.7\% DOC, $0.5 \mathrm{M} \mathrm{LiCl}$, freshly added protease inhibitor) 
for 5 times followed by wash with TE once. One hundred microliters of elution buffer (50 $\mathrm{mM}$ Tris- $\mathrm{HCl} \mathrm{pH}$ 8.0, $1 \mathrm{mM}$ EDTA, 1\% SDS) was added and beads were incubated in Thermo mixer at $65^{\circ} \mathrm{C}$ for 30 min with max speed. Supernatant was collected and treated with protease $\mathrm{K}$ at $55^{\circ} \mathrm{C}$ for $2 \mathrm{~h}$, then purified with DNA purification kit (Tiangen). RNA was removed by treatment of RNase at $37^{\circ} \mathrm{C}$ for $1 \mathrm{~h}$. DNA was then purified by AMPure Beads (Beckman) and subjected to DNA library preparation as described below.

\section{RNA-seq library preparation and sequencing}

Five milligrams of RNA was extracted using quick-RNA MiniPrep kit (Zymo) and then treated with DNase I (Fermentas) at $37^{\circ} \mathrm{C}$ for $1 \mathrm{~h}$. RNA was then purified using AMPure beads. Poly-A tailed mRNA was collected using Dynabeads ${ }^{\mathrm{Th}}$ mRNA purification kit (Invitrogen). Purified RNA was fragmented with RNA Fragmentation Buffer (NEB) at $95^{\circ} \mathrm{C}$ for $5 \mathrm{~min}$. Reaction was stopped and RNA was purified by AMPure beads. First-strand cDNA was synthesized with a commercial kit using both oligo dT and random primers (Invitrogen). Second-strand cDNA was synthesized with secondstrand synthesis buffer (Invitrogen), $\mathrm{MgCl}_{2}$, DTT, dNTP, dUTP, RNase H (Fermentas), E. coli DNA ligase (NEB), and DNA polymerase I (NEB). DNA was purified after $2 \mathrm{~h}$ incubation on thermomixer at $16^{\circ} \mathrm{C}$. Synthesized cDNA was subjected to DNA library preparation as described below.

\section{Library construction}

Purified DNA or cDNA was subjected to NEBNext Ultra II DNA Library Prep Kit for Illumina (NEB). The DNA was resuspended in $25 \mu \mathrm{lddH} 2 \mathrm{O}$, followed by end-repair/A-tailing with $3.5 \mu \mathrm{l}$ End Prep buffer and $1.5 \mu \mathrm{l}$ enzyme mix according to manufactory instruction. The ligation reaction was then performed by adding diluted $1.25 \mu \mathrm{l}$ adaptors (NEB), $15 \mu \mathrm{l}$ Ligation master mix, $0.5 \mu \mathrm{l}$ Ligation enhancer, at $4{ }^{\circ} \mathrm{C}$ for overnight. The ligation reaction was treated with $1.5 \mu \mathrm{l}$ USERTM enzyme according to the instruction and was purified by AMPure beads. The 1st round PCR was performed by adding $25 \mu \mathrm{l} 2 \mathrm{x}$ KAPA HiFi HotStart Ready Mix (KAPA biosystems) with primers of NEB Oligos kit, with the program of $98^{\circ} \mathrm{C}$ for $45 \mathrm{~s},\left(98^{\circ} \mathrm{C}\right.$ for $15 \mathrm{~s}$ and $60^{\circ} \mathrm{C}$ for $10 \mathrm{~s}$ ) with $8-9$ cycles and $72^{\circ} \mathrm{C}$ for $1 \mathrm{~min}$. DNA was purified using 1x AMPure beads and a 2nd round PCR was performed with $25 \mu \mathrm{l} 2 \mathrm{x}$ KAPA HiFi HotStart Ready Mix with Illumina universal primers, and same PCR program for 8 cycles. The final libraries were purified by $1 \mathrm{x}$ AMPure beads and subjected to nextgeneration sequencing. All libraries were sequenced on Illumina HiSeq 2500 or HiSeq XTen according to the manufacturer's instruction.

\section{sisHi-C library generation and sequencing}

The sisHi-C library generation was performed as described previously [41]. Briefly, spermatogenetic cells were fixed with $1 \%$ formaldehyde at room temperature (RT) for $10 \mathrm{~min}$. Formaldehyde was quenched with glycine for $10 \mathrm{~min}$ at RT. Cells were washed with 1XPBS for two times and then lysed in $50 \mu$ lysis buffer $(10 \mathrm{mM}$ Tris- $\mathrm{HCl}$ $\mathrm{pH} 7.4,10 \mathrm{mM} \mathrm{NaCl}, 0.1 \mathrm{mM}$ EDTA, $0.5 \% \mathrm{NP}-40$ and proteinase inhibitor) on ice for $50 \mathrm{~min}$. After spinning at $3000 \mathrm{rpm} / \mathrm{min}$ in $4{ }^{\circ} \mathrm{C}$ for $5 \mathrm{~min}$, the supernatant was discarded with a pipette carefully. Chromatin was solubilized in $0.5 \%$ SDS and incubated at $62{ }^{\circ} \mathrm{C}$ for $10 \mathrm{~min}$. SDS was quenched by $10 \%$ Triton $\mathrm{X}-100$ at $37^{\circ} \mathrm{C}$ for 
$30 \mathrm{~min}$. Then, the nuclei were digested with $50 \mathrm{U}$ Mbo I at $37^{\circ} \mathrm{C}$ overnight with rotation. Mbo I was then inactivated at $62{ }^{\circ} \mathrm{C}$ for $20 \mathrm{~min}$. To fill in the biotin to the DNA, dATP, dGTP, dTTP, biotin-14-dCTP, and Klenow were added to the solution and the reaction was carried out at $37^{\circ} \mathrm{C}$ for $1.5 \mathrm{~h}$ with rotation. The fragments were ligated at RT for $6 \mathrm{~h}$ with rotation. This was followed by reversal of crosslink and DNA purification. DNA was sheared to 300-500 bp with Covaris M220. The biotin-labeled DNA was then pulled down with 10ul Dynabeads MyOne Streptavidin C1 (Life Technology). Sequencing library preparation was performed on beads, including end-repair, dATP tailing and adaptor-ligation. DNA was eluted twice by adding $20 \mathrm{ul}$ water to the tube and incubation at $66^{\circ} \mathrm{C}$ for $20 \mathrm{~min}$. Nine to 15 cycles of PCR amplification were performed with Extaq (Takara). Finally, size selection was done with AMPure XP beads and fragments ranging from $200 \mathrm{bp}$ to $1000 \mathrm{bp}$ were selected. All the libraries were sequenced on Illumina HiSeq2500 or HiSeq Xten according to the manufacturer's instruction.

\section{Imaging data analysis}

The maximum projection of $\mathrm{Z}$ stack was used for the quantification of cell numbers. The Imaris 9.0.1platfrom was used to process the HP1apha and H3K9me3 channel intensity simultaneously and to measure the degree of colocolization of two channel using Coloc2. Analysis trajectory of live cell loci site using MTrack2 Plugins. Fluorescent puncta were identified as local maxima satisfying the minimum intensity and $\mathrm{min} /$ max peak with threshold by Gaussian fitting. Data are represented as mean \pm SEM. The exact number, $n$, of data points and the representation of $n$ (cells, independent experiments) are indicated in the respective figure legends and in the "Results" section.

\section{ChIP data processing}

The paired-end ChIP reads were aligned with the parameters: $-\mathrm{t}-\mathrm{q}-\mathrm{N} 1-\mathrm{L} 25-\mathrm{X}$ 1000 --no-mixed --no-discordant. All unmapped reads, non-uniquely mapped reads, reads with low mapping quality (MAPQ $<20$ ), and PCR duplicates were removed. For downstream analysis, we normalized the read counts by computing the numbers of reads per kilobase of bin per million of reads sequenced (RPKM) for 100-bp bins of the genome. To minimize the batch and cell type variation, RPKM values across whole genome were further $Z$-score normalized. To visualize the ChIP signals in the UCSC genome browser, we generated the RPKM values on a $100 \mathrm{bp}$-window base. H3K9me3 peaks were called using MACS2 [42] with the parameters - $\mathrm{g}$ hs -broad -nomodel nolambda --broad and noisy peaks with very weak signals $($ RPKM $<5)$ were removed from further analyses. Adjacent peaks with very close distance $(<5 \mathrm{~kb})$ were merged for downstream analyses.

\section{RNA-seq data processing}

All RNA-seq data were mapped to hg19 reference genome by Tophat. The gene expression levels were calculated by Cufflinks (version 2.2.1) using the refFlat database from the UCSC genome browser. 


\section{Hi-C data mapping}

Paired end raw reads of $\mathrm{Hi}-\mathrm{C}$ libraries were aligned, processed, and iteratively corrected using HiC-Pro (version 2.7.1b) as described [43]. Briefly, sequencing reads were first independently aligned to the human reference genome (hg19) using the bowtie2 end-toend algorithm and "-very-sensitive" option. To rescue the chimeric fragments spanning the ligation junction, the ligation site was detected and the $5^{\prime}$ fraction of the reads was aligned back to the reference genome. Unmapped reads, multiple mapped reads, and singletons were then discarded. Pairs of aligned reads were then assigned to Mbo I restriction fragments. Read pairs from uncut DNA, self-circle ligation, and PCR artifacts were filtered out and the valid read pairs involving two different restriction fragments were used to build the contact matrix. Valid read pairs were then binned at a specific resolution by dividing the genome into bins of equal size. We chose 100-kb bin size for examination of global interaction patterns of the whole chromosome and 40-kb bin size to show local interactions and to perform TAD calling. Then, the binned interaction matrices were normalized using the iterative correction method $[43,44]$ to correct the biases such as GC content, mappability, and effective fragment length in $\mathrm{Hi}-\mathrm{C}$ data.

\section{Identification of chromatin compartments A/B}

Conventional chromatin compartments A and B were identified with a method described previously [45] with some modifications. The normalized $100 \mathrm{~kb}$ interaction matrices for each stage were used in this analysis. Firstly, the bins that have no interactions with any other bins were removed before expected interaction matrices were calculated. Observed/ expected matrices were generated using a sliding window approach [31] with the bin size of $400 \mathrm{~kb}$ and the step size of $100 \mathrm{~kb}$. Principal component analysis was performed on the correlation matrices generated from the observed/expected matrices. The first principal component of the correlation matrices together with gene density were used to identify A/B compartments. In this analysis, the correlation matrices were calculated according to the interaction matrices separated according to the location of predicted centromeres for each chromatin, as the principal component often reflects the partitioning of chromosome arms [45]. Juicebox was used to generate all chromosome-wide as well as the zoom-in views of interaction frequency heatmap in this study.

\section{Analysis of the promoter H3K4me3 and H3K27ac enrichment}

Annotated promoters (TSS $\pm 2.5 \mathrm{~kb}$ ) of the hg19 refFlat annotation database from the USCS genome browser were used. Whole genome Z-score normalized RPKM scores were used for calculation. The nearest genes to the sgRNA target were found by bedtools (-closest).

\section{Supplementary Information}

Supplementary information accompanies this paper at https://doi.org/10.1186/s13059-020-02201-1.

Additional file 1: Figure S1. H3K9 trimethylation of $\mathrm{C} 19 \mathrm{Q}$ regions mediated by the EpiGo-KRAB system. (A) H3K9me3 states of C19Q region in the cell lines of U2OS wildtype (WT), U2OS-HP1a-HaloTag (HP1a), EpiGo-Control (dCas9) or EpiGo-KRAB (dCas9-KRAB). ChIP-seq of H3K9me3 was performed in these four cell lines. C19Q repeats shown the C19Q target sites. (B) C19Q-1 region is chosen to show the difference of H3K9me3 state between U2OS wildtype (WT), U2OS-HP1a-HaloTag (HP1a), EpiGo-Control (dCas9) or EpiGo-KRAB (dCas9-KRAB) cell lines. C19Q repeats shown the $\mathrm{C} 19 \mathrm{Q}$ target sites. Figure S2. Negative correlation of H3K9 trimethylation and active transcription. The H3K9me3 state and RNA levels of 30-40 Mb regions in chromosome 1-6 were shown in A-F respectively. The ChIP-seq of 
fold change. (B) Plot showing the relationship between distance (CRISPR to the nearest TSS) and promoter H3K4me3 enrichment change. (C) Plot showing the relationship between distance (CRISPR to the nearest TSS) and promoter H3K27ac enrichment change.

Additional file 2: Table S1. Target sites of C19Q on the q-arm of human chromosome 19.

Additional file 3: Table S2. A list of regions and primers for Oligopaint FISH probes in This Study.

Additional file 4: Table S3. A list of template oligonucleotides for Oligopaint FISH probes in This Study.

Additional file 5: Movie S1. Tracking the movement of C19Q loci in U2OS-EpiGo-Control cells. Images were cropped to 50X50 pixels and each video includes 300 frames (a total time of 30 seconds). The imaging rate is 100 milliseconds per frame and the play rate is 30 frames per second. The individual locus movements were corrected for the possible movements of microscope stage.

Additional file 6: Movie S2. Tracking the movement of C19Q loci in U2OS-EpiGo-KRAB cells. The image processing details are the same as described in the Video $\mathbf{S 1}$.

Additional file 7. Review history.

\section{Acknowledgements}

We thank Luke Lavis (Janelia Research Campus, Howard Hughes Medical Institute, Ashburn, VA, USA) for the HaloTag JF-549. U2OS Genomic DNA was a gift Xingxu Huang. We thank Jianan Li for help with Lentivirus production. We are grateful to Guisheng Zhong, Cuiping Tian, Xiaoming Li, and Yi Qian for help with imaging. We thank Pengwei Zhang and Shuangli Zhang for help with cell sorting. OMX4 microscopy was provided by Shanghai Institute for Advanced Immunochemical Studies (SIAIS) at Shanghaitech University and Fluorescence activated cell sorting (FACS) was provided by iHuman Institute of ShanghaiTech. We thank members of the Ma's laboratory and Xie's laboratory for their comments during preparation of the manuscript.

\section{Peer review information}

Yixin Yao and Kevin Pang were the primary editors of this article and managed its editorial process and peer review in collaboration with the rest of the editorial team.

\section{Review history}

The review history is available as Additional file 7.

\section{Authors' contributions}

H.M. conceived this project. H.M. and Y.F. designed the experiments. A.N. and S.Z. performed the data mining of chromosome-specific repeats. C.Y. made the constructs and performed qPCR. X.W. and X.H. performed the Oligopaint FISH and ImmunoFISH. Y.F. performed fluorescence imaging and 3D-SIM. Y.W. performed the RNA-seq, ChIP-seq, and Hi-C. H.M., Y.F., W.X., Y.W., J.Z., X.X., and T.P. interpreted the data. Y.F., Y.W., W.X., and H.M. wrote the paper with input from all the authors. The authors read and approved the final manuscript.

\section{Funding}

This work was funded by National Natural Science Foundation of China (No. 31970591 to H. Ma) and the Shanghai Pujiang program (19PJ1408000 to H. Ma). The initial phase of this work by H.M. in the laboratory of T.P. was funded in part through the U.S. National Institutes of Health 4D Nucleome Initiative, grant U01-DA-040588 to Paul Kaufman and T.P. This study was also funded by the National Natural Science Foundation of China (31988101, 31830047, 31725018 to W. X.), the THU-PKU Center for Life Sciences (W. X.), the Beijing Municipal Science \& Technology Commission (grant Z181100001318006 to W. X.). W. X. is an HHMl international research scholar.

\section{Availability of data and materials}

Raw sequence reads of U2OS-EpiGo-Control and U2OS-EpiGo-KRAB experiments are deposited in NCBI GEO: GSE137469 [46].

\section{Competing interests}

The authors declare no competing interests.

\section{Author details}

${ }^{1}$ School of Biotechnology, East China University of Science and Technology, Shanghai, China; School of Life Science and Technology, ShanghaiTech University, Shanghai, China. ${ }^{2}$ Center for Stem Cell Biology and Regenerative Medicine, MOE Key Laboratory of Bioinformatics, School of Life Sciences, Tsinghua University, Beijing, China. ${ }^{3}$ Tsinghua-Peking Center for Life Sciences, Beijing, China. ${ }^{4}$ School of Life Science and Technology, ShanghaiTech University, Beijing, China. ${ }^{5}$ School of Pharmacy, East China University of Science and Technology, Shanghai, China. ${ }^{6}$ Department of Computer Science, University of Central Florida, Orlando, FL, USA. ${ }^{7}$ Department of Biochemistry and Molecular Pharmacology, University of Massachusetts Medical School, Worcester, MA, USA. ${ }^{8}$ School of Biotechnology East China University of Science and Technology, Shanghai, China.

Received: 29 May 2020 Accepted: 9 November 2020 Published online: 08 December 2020

\section{References}

1. Dixon JR, Selvaraj S, Yue F, Kim A, Li Y, Shen Y, Hu M, Liu JS, Ren B. Topological domains in mammalian genomes identified by analysis of chromatin interactions. Nature. 2012;485:376-80. 
2. de Laat W, Duboule D. Topology of mammalian developmental enhancers and their regulatory landscapes. Nature. 2013:502:499-506.

3. Sima J, Chakraborty A, Dileep V, Michalski M, Klein KN, Holcomb NP, Turner JL, Paulsen MT, Rivera-Mulia JC, TrevillaGarcia C, et al. Identifying cis elements for spatiotemporal control of mammalian DNA replication. Cell. 2019;176:816-30 e818.

4. Gibcus JH, Dekker J. The hierarchy of the 3D genome. Mol Cell. 2013:49:773-82.

5. Nuebler J, Fudenberg G, Imakaev M, Abdennur N, Mirny LA. Chromatin organization by an interplay of loop extrusion and compartmental segregation. Proc Natl Acad Sci U S A. 2018;115:E6697-706.

6. Stadhouders R, Filion GJ, Graf T. Transcription factors and 3D genome conformation in cell-fate decisions. Nature. 2019; 569:345-54.

7. Larson AG, Elnatan D, Keenen MM, Trnka MJ, Johnston JB, Burlingame AL, Agard DA, Redding S, Narlikar GJ. Liquid droplet formation by HP1alpha suggests a role for phase separation in heterochromatin. Nature. 2017;547:236-40.

8. Strom AR, Emelyanov AV, Mir M, Fyodorov DV, Darzaca X, Karpen GH. Phase separation drives heterochromatin domain formation. Nature. 2017;547:241-5.

9. Wang L, Gao Y, Zheng X, Liu C, Dong S, Li R, Zhang G, Wei Y, Qu H, Li Y, et al. Histone modifications regulate chromatin compartmentalization by contributing to a phase separation mechanism. Mol Cell. 2019;76:646-59 e646.

10. Falk M, Feodorova Y, Naumova N, Imakaev M, Lajoie BR, Leonhardt H, Joffe B, Dekker J, Fudenberg G, Solovei I, Mirny LA. Heterochromatin drives compartmentalization of inverted and conventional nuclei. Nature. 2019;570:395-9.

11. Rao SS, Huntley MH, Durand NC, Stamenova EK, Bochkov ID, Robinson JT, Sanborn AL, Machol I, Omer AD, Lander ES, Aiden EL. A 3D map of the human genome at kilobase resolution reveals principles of chromatin looping. Cell. 2014 159:1665-80

12. Wang S, Su JH, Beliveau BJ, Bintu B, Moffitt JR, Wu CT, Zhuang X. Spatial organization of chromatin domains and compartments in single chromosomes. Science. 2016;353:598-602.

13. Bintu B, Mateo LJ, Su JH, Sinnott-Armstrong NA, Parker M, Kinrot S, Yamaya K, Boettiger AN, Zhuang X. Super-resolution chromatin tracing reveals domains and cooperative interactions in single cells. Science. 2018;362:eaau1783.

14. Mateo LJ, Murphy SE, Hafner A, Cinquini IS, Walker CA, Boettiger AN. Visualizing DNA folding and RNA in embryos at single-cell resolution. Nature. 2019;568:49-54.

15. Nir G, Farabella I, Perez Estrada C, Ebeling CG, Beliveau BJ, Sasaki HM, Lee SD, Nguyen SC, McCole RB, Chattoraj S, et al. Walking along chromosomes with super-resolution imaging, contact maps, and integrative modeling. PLoS Genet. 2018; 14:e1007872.

16. Cardozo Gizzi AM, Cattoni DI, Fiche JB, Espinola SM, Gurgo J, Messina O, Houbron C, Ogiyama Y, Papadopoulos GL, Cavalli $\mathrm{G}$, et al. Microscopy-based chromosome conformation capture enables simultaneous visualization of genome organization and transcription in intact organisms. Mol Cell. 2019;74:212-22 e215.

17. Ma H, Tu LC, Naseri A, Chung YC, Grunwald D, Zhang S, Pederson T. CRISPR-Sirius: RNA scaffolds for signal amplification in genome imaging. Nat Methods. 2018;15:928-31.

18. Chen B, Gilbert LA, Cimini BA, Schnitzbauer J, Zhang W, Li GW, Park J, Blackburn EH, Weissman JS, Qi LS, Huang B. Dynamic imaging of genomic loci in living human cells by an optimized CRISPR/Cas system. Cell. 2013;155:1479-91.

19. Thakore PI, D'Ippolito AM, Song L, Safi A, Shivakumar NK, Kabadi AM, Reddy TE, Crawford GE, Gersbach CA. Highly specific epigenome editing by CRISPR-Cas9 repressors for silencing of distal regulatory elements. Nat Methods. 2015;12: $1143-9$.

20. Klann TS, Black JB, Chellappan M, Safi A, Song L, Hilton IB, Crawford GE, Reddy TE, Gersbach CA. CRISPR-Cas9 epigenome editing enables high-throughput screening for functional regulatory elements in the human genome. Nat Biotechnol. 2017;35:561-8.

21. Ma H, Tu LC, Naseri A, Huisman M, Zhang S, Grunwald D, Pederson T. Multiplexed labeling of genomic loci with dCas9 and engineered sgRNAs using CRISPRainbow. Nat Biotechnol. 2016;34:528-30.

22. Frietze S, O'Geen H, Blahnik KR, Jin VX, Farnham PJ. ZNF274 recruits the histone methyltransferase SETDB1 to the $3^{\prime}$ ends of ZNF genes. PLoS One. 2010;5:e15082.

23. Schultz DC, Ayyanathan K, Negorev D, Maul GG, Rauscher FJ 3rd. SETDB1: a novel KAP-1-associated histone H3, lysine 9specific methyltransferase that contributes to HP1-mediated silencing of euchromatic genes by KRAB zinc-finger proteins. Genes Dev. 2002;16:919-32.

24. Groner AC, Meylan S, Ciuffi A, Zangger N, Ambrosini G, Denervaud N, Bucher P, Trono D. KRAB-zinc finger proteins and KAP1 can mediate long-range transcriptional repression through heterochromatin spreading. PLoS Genet. 2010;6:e1000869.

25. Keenen MM, Larson AG, Narlikar GJ, Redding S. Dissecting the mechanism of HP1 mediated chromatin compaction via single molecule DNA curtains. Biophysical J. 2018;114:30a.

26. Leopold K, Stirpe A, Schalch T. Transcriptional gene silencing requires dedicated interaction between HP1 protein Chp2 and chromatin remodeler Mit1. Genes Dev. 2019;33:565-77.

27. Gauchier M, Kan S, Barral A, Sauzet S, Agirre E, Bonnell E, Saksouk N, Barth TK, Ide S, Urbach S, et al. SETDB1-dependent heterochromatin stimulates alternative lengthening of telomeres. Sci Adv. 2019;5:eaav3673.

28. Fortin JP, Hansen KD. Reconstructing A/B compartments as revealed by $\mathrm{Hi}-\mathrm{C}$ using long-range correlations in epigenetic data. Genome Biol. 2015;16:180.

29. Zhang K, Wu DY, Zheng H, Wang Y, Sun QR, Liu X, Wang LY, Xiong WJ, Wang Q, Rhodes JDP, et al. Analysis of genome architecture during SCNT reveals a role of cohesin in impeding minor ZGA. Mol Cell. 2020;79:234-50 e239.

30. Barutcu AR, Maass PG, Lewandowski JP, Weiner CL, Rinn JL. A TAD boundary is preserved upon deletion of the CTCFrich Firre locus. Nat Commun. 2018;9:1444.

31. Dixon JR, Jung I, Selvaraj S, Shen Y, Antosiewicz-Bourget JE, Lee AY, Ye Z, Kim A, Rajagopal N, Xie W, et al. Chromatin architecture reorganization during stem cell differentiation. Nature. 2015;518:331-6.

32. van Steensel B, Belmont AS. Lamina-associated domains: links with chromosome architecture, heterochromatin, and gene repression. Cell. 2017;169:780-91.

33. Meuleman W, Peric-Hupkes D, Kind J, Beaudry JB, Pagie L, Kellis M, Reinders M, Wessels L, van Steensel B. Constitutive nuclear lamina-genome interactions are highly conserved and associated with ATT-rich sequence. Genome Res. 2013;23: 270-80. 
34. Erdel F, Rademacher A, Vlijm R, Tunnermann J, Frank L, Weinmann R, Schweigert E, Yserentant K, Hummert J, Bauer C, et al. Mouse heterochromatin adopts digital compaction states without showing hallmarks of HP1-driven liquid-liquid phase separation. Mol Cell. 2020;78:236-49 e237.

35. Kraushaar DC, Zhao K. The epigenomics of embryonic stem cell differentiation. Int J Biol Sci. 2013;9:1134-44.

36. Altun G, Loring JF, Laurent LC. DNA methylation in embryonic stem cells. J Cell Biochem. 2010;109:1-6.

37. Miyamoto T, Furusawa C, Kaneko K. Pluripotency, differentiation, and reprogramming: a gene expression dynamics model with epigenetic feedback regulation. PLoS Comput Biol. 2015;11:e1004476.

38. Ma H, Naseri A, Reyes-Gutierrez P, Wolfe SA, Zhang S, Pederson T. Multicolor CRISPR labeling of chromosomal loci in human cells. Proc Natl Acad Sci U S A. 2015;112:3002-7.

39. Marcais G, Kingsford C. A fast, lock-free approach for efficient parallel counting of occurrences of k-mers. Bioinformatics. 2011;27:764-70.

40. Beliveau BJ, Boettiger AN, Nir G, Bintu B, Yin P, Zhuang X, Wu CT. In situ super-resolution imaging of genomic DNA with OligoSTORM and OligoDNA-PAINT. Methods Mol Biol. 2017;1663:231-52.

41. Du Z, Zheng H, Huang B, Ma R, Wu J, Zhang X, He J, Xiang Y, Wang Q, Li Y. Allelic reprogramming of 3D chromatin architecture during early mammalian development. Nature. 2017;547:232.

42. Zhang Y, Liu T, Meyer CA, Eeckhoute J, Johnson DS, Bernstein BE, Nusbaum C, Myers RM, Brown M, Li W, Liu XS. Modelbased analysis of ChIP-Seq (MACS). Genome Biol. 2008;9:R137.

43. Servant N, Varoquaux N, Lajoie BR, Viara E, Chen CJ, Vert JP, Heard E, Dekker J, Barillot E. HiC-Pro: an optimized and flexible pipeline for $\mathrm{Hi}-\mathrm{C}$ data processing. Genome Biol. 2015;16:259.

44. Imakaev M, Fudenberg G, McCord RP, Naumova N, Goloborodko A, Lajoie BR, Dekker J, Mirny LA. Iterative correction of Hi-C data reveals hallmarks of chromosome organization. Nat Methods. 2012;9:999-1003.

45. Lieberman-Aiden E, van Berkum NL, Williams L, Imakaev M, Ragoczy T, Telling A, Amit I, Lajoie BR, Sabo PJ, Dorschner $\mathrm{MO}$, et al. Comprehensive mapping of long-range interactions reveals folding principles of the human genome. Science. 2009;326:289-93.

46. Ying Feng, Yao Wang, Xiangnan Wang, Xiaohui He, Chen Yang, Ardalan Naseri, Thoru Pederson, Jing Zheng, Shaojie Zhang, Xiao Xiao, Wei Xie, and Hanhui Ma.Datasets. Gene Expression Omnibus. Epigenetic perturbation and genome imaging reveal distinct roles of H3K9me3 in chromatin architecture and transcription (2020). https://www.ncbi.nIm.nih. gov/geo/query/acc.cgi?acc=GSE137469.

\section{Publisher's Note}

Springer Nature remains neutral with regard to jurisdictional claims in published maps and institutional affiliations.

Ready to submit your research? Choose BMC and benefit from:

- fast, convenient online submission

- thorough peer review by experienced researchers in your field

- rapid publication on acceptance

- support for research data, including large and complex data types

- gold Open Access which fosters wider collaboration and increased citations

- maximum visibility for your research: over $100 \mathrm{M}$ website views per year

At $B M C$, research is always in progress.

Learn more biomedcentral.com/submissions 